\title{
Rank-Based Extensions of the Brock, Dechert, and Scheinkman Test
}

\author{
Christian GENEST, Kilani GHOUDI, and Bruno RÉMILLARD
}

\begin{abstract}
This article proposes new tests of randomness for innovations in a large class of time series models. These tests are based on functionals of empirical processes constructed from either the model residuals or their associated ranks. The asymptotic behavior of these processes is determined under the null hypothesis of randomness. The limiting distributions are seen to be independent of estimation errors under appropriate regularity conditions. Several test statistics are derived from these processes; the classical Brock, Dechert, and Scheinkman statistic and a rank-based analog are included as special cases. Because the limiting distributions of the rank-based test statistics are marginfree, their finite-sample $p$ values can be easily calculated by simulation. Monte Carlo experiments show that these statistics are quite powerful against several classes of alternatives.
\end{abstract}

KEY WORDS: Brock, Dechert, and Scheinkman statistic; Copula; Empirical process; Pseudo-observation; Randomness; Rank; Time series.

\section{INTRODUCTION}

Univariate time series models involve error terms $\varepsilon_{i}$ that are typically assumed to be mutually independent with common distribution function $F$. An important step in validating such models is to test the hypothesis of randomness for the sequence $\left(\varepsilon_{i}\right)$. Usually, the weaker hypothesis $H_{0}=H_{0}(m)$ of independence of $m$ consecutive innovations is tested for fixed integer $m$. This is the problem that we consider here.

When the parameters of the model are known, the innovations can be observed, and various tools are available for testing $H_{0}$. Common procedures are based on autocorrelations (Moran 1948; Ljung and Box 1978; Dufour and Roy 1985; Hong 2000), entropy measures (Robinson 1991; Hong and White 2005), rank-based dependence measures (Hallin, Ingenbleek, and Puri 1985, 1987; Hallin and Puri 1992; Ferguson, Genest, and Hallin 2000), empirical distribution functions (Skaug and Tjøstheim 1993; Delgado 1996; Ghoudi, Kulperger, and Rémillard 2001), empirical characteristic functions (Hong 1999; Bilodeau and Lafaye de Micheaux 2005), and empirical copulas (Genest and Rémillard 2004).

In reality, the model parameters are usually unknown, and the $\varepsilon_{i}$ 's are then unobservable. Thus a test for randomness must be based on "residuals," $e_{i}$. These are typically computed by plugging in the estimated parameters in an equation relating $\varepsilon_{i}$ and the observed data $y_{i}$ at time $i \in\{1, \ldots, n\}$. This equation also may depend on finitely many previous values $y_{i-1}, \ldots, y_{i-p}$ and $\varepsilon_{i-1}, \ldots, \varepsilon_{i-q}$. A major stumbling block associated with this approach is that the asymptotic distribution of the test statistic generally will depend on both the unknown parameter values and the (infinite-dimensional) nuisance parameter $F$. Authors who have dealt with this thorny issue include Hallin and Puri (1994) and Hallin and Jurečková (1999).

One ingenious way around this problem is provided by the statistic of Brock, Dechert, and Scheinkman (BDS), the limiting behavior of which under $H_{0}$ was shown by Brock, Dechert,

Christian Genest is Professor, Département de mathématiques et de statistique, Université Laval, Québec, Québec, Canada G1K 7P4 (E-mail: Christian. Genest@mat.ulaval.ca). Kilani Ghoudi is Professor, United Arab Emirates University, Al Ain, United Arab Emirates (E-mail: kghoudi@uaeu.ac.ae) Bruno Rémillard is Professor, Service de l'enseignement des méthodes quantitatives de gestion, HEC Montréal, Montréal, Québec, Canada H3T 2A7 (E-mail: Bruno.Remillard@hec.ca). Partial funding in support of this work was provided by the Natural Sciences and Engineering Research Council of Canada, by the Fonds québécois de la recherche sur la nature et les technologies, and by the Institut de finance mathématique de Montréal.
LeBaron, and Scheinkman (1996) to be the same whether the model parameters are known or estimated in a $n^{1 / 2}$-consistent fashion. The BDS statistic $S_{n, \delta}$ of embedding dimension $m$ is based on a comparison of the observed and expected numbers of pairs of vectors $\mathbf{w}_{i}=\left(w_{i 1}, \ldots, w_{i m}\right)=\left(e_{i}, \ldots, e_{i+m-1}\right)$ for which $\left\|\mathbf{w}_{i}-\mathbf{w}_{j}\right\|=\max _{k \in\{1, \ldots, m\}}\left|w_{i k}-w_{j k}\right| \leq \delta$ for some chosen proximity parameter $\delta>0$. Rejection of $H_{0}$ occurs when $\left|S_{n, \delta}\right|$ is too large, by reference to its asymptotic null distribution.

Letting $e_{i+n}=e_{i}$ for all $i \in \mathbb{N}$, we can define a circular version of the BDS statistic by

$$
S_{n, \delta}\left(e_{1}, \ldots, e_{n+m-1}\right)=n^{1 / 2}\left\{S_{m}(\delta)-S(\delta)^{m}\right\} / \sigma_{n}(\delta),
$$

where

$$
\begin{aligned}
S_{m}(\delta) & =\frac{1}{\left(\begin{array}{l}
n \\
2
\end{array}\right)} \sum_{1 \leq i<j \leq n} \mathbf{1}\left(\left\|\mathbf{w}_{i}-\mathbf{w}_{j}\right\| \leq \delta\right), \\
S(\delta) & =\frac{1}{\left(\begin{array}{l}
n \\
2
\end{array}\right)} \sum_{1 \leq i<j \leq n} \mathbf{1}\left(\left|e_{j}-e_{i}\right| \leq \delta\right),
\end{aligned}
$$

and $\sigma_{n}(\delta)$ is an estimate of the standard deviation of $n^{1 / 2} \times$ $\left\{S_{m}(\delta)-S(\delta)^{m}\right\}$. The specific estimator proposed by Brock et al. (1996) is

$$
\begin{array}{r}
\sigma_{n}^{2}(\delta)=4\left\{\tau_{n}(\delta)^{m}-S(\delta)^{2 m}\right\}-4 m^{2} S(\delta)^{2 m-2}\left\{\tau_{n}(\delta)-S(\delta)^{2}\right\} \\
+8 \sum_{k=1}^{m-1} S(\delta)^{2 k}\left\{\tau_{n}(\delta)^{m-k}-S(\delta)^{2 m-2 k}\right\},
\end{array}
$$

where

$$
\tau_{n}(\delta)=n^{-3} \sum_{i=1}^{n} \sum_{j=1}^{n} \sum_{k=1}^{n} \mathbf{1}\left(\left|e_{j}-e_{i}\right| \leq \delta\right) \mathbf{1}\left(\left|e_{j}-e_{k}\right| \leq \delta\right) .
$$

Brock et al. (1996) showed that for a wide class of time series models, the large-sample distribution of $S_{n, \delta}$ is standard normal under the null hypothesis of randomness, just as it would be if the model parameters were known and the statistic were computed from the $\varepsilon_{i}$, which then would be directly observable.

Along with its strengths, the BDS procedure suffers from three major weaknesses. First, there is arbitrariness in the choice of $\delta$, which may affect both the power and size of

() 2007 American Statistical Association Journal of the American Statistical Association December 2007, Vol. 102, No. 480, Theory and Methods DOI 10.1198/016214507000001076 
the test. In practice, Brock et al. (1996) recommended taking $\delta \in[\hat{\sigma} / 2,3 \hat{\sigma} / 2]$, where $\hat{\sigma}$ is the standard deviation of the pseudosample $e_{1}, \ldots, e_{n+m-1}$. Another option, considered by Kočenda (2001), is to base the test on the estimate of the slope of $\log \left\{S_{m}(\delta)\right\}$ with respect to $\log (\delta)$. This slope is computed over a range of values of $\delta$ that Kočenda and Briatka (2005) have optimized.

A second limitation of the BDS test is that the probability of rejecting $H_{0}$ does not always approach 1 as $n \rightarrow \infty$ under the alternative. Models exist under which the expected value of the test statistic vanishes for at least one (and possibly all) $\delta$.

The third (and most critical) difficulty associated with the BDS test is that although the statistic converges to a standard normal distribution under $H_{0}$, this convergence is sometimes very slow, depending on the choice of $\delta$ and $F$ (see, e.g., tables A.5-A.6 in Brock, Hsieh, and LeBaron 1991). This is inconvenient because neither the level nor the power of the test can be determined precisely unless $F$ is known. Whereas critical points or $p$ values could be found in the latter case (e.g., adapting Algorithm 1), the rate of convergence varies considerably from one choice of $F$ to another (see, e.g., Brock et al. 1996).

In this article extensions of the BDS statistic are considered that are freed from either some or all of these limitations. The first alternative procedure, considered in Section 2, is a rankbased equivalent of the original BDS statistic. Although it depends on $\delta$, its asymptotic distribution is independent of $F$ (Thm. 1) and its finite-sample distribution can be simulated easily (Algorithm 1); therefore, the speed at which the test statistic converges in law is not an issue.

In Section 3 empirical processes extending $S_{n, \delta}$ are proposed and their asymptotic behavior is studied (Thm. 2); rank-based equivalents are also considered (Thm. 3). This leads in Section 4 to tests of randomness whose statistics are free of $\delta$ and whose asymptotic distributions are independent of the model parameters; algorithms approximating their null distribution are provided. The finite-sample performance of the proposed statistics is considered in Section 5, with power estimated by simulation for a wide range of serial dependence alternatives. This is followed by a discussion of the relative merits of the various tests. Small illustrations of the methodology are treated in Section 6, and the relevance of technical conditions on $F$ required to obtain the asymptotic results is addressed in Section 7. All proofs are relegated to the Appendixes.

\section{A RANK-BASED VERSION OF THE BDS STATISTIC}

Given residuals $e_{1}, \ldots, e_{n}$ from a time series model, let $\tilde{e}_{i}=$ $\operatorname{rank}\left(e_{i}\right) /(n+1)$ be their normalized ranks and write $\tilde{e}_{i+n}=\tilde{e}_{i}$ for every integer $i \geq 1$. A nonparametric analog of the BDS statistic (1) is

$$
\tilde{S}_{n, \delta}=S_{n, \delta}\left(\tilde{e}_{1}, \ldots, \tilde{e}_{n+m-1}\right) .
$$

The asymptotic normality of this statistic depends critically on Assumptions I and II. As shown in Section 7, these assumptions are met for, say, linear and nonlinear $\operatorname{AR}(p)$ as well as standard $\operatorname{ARMA}(p, q)$ models. For $\mathbf{x}=\left(x_{1}, \ldots, x_{m}\right) \in[-\infty, \infty]^{m}$, let $K(\mathbf{x})=F\left(x_{1}\right) \cdots F\left(x_{m}\right)$ and define

$$
K_{n}(\mathbf{x})=n^{-1} \sum_{i=1}^{n} \mathbf{1}\left(\mathbf{w}_{i} \leq \mathbf{x}\right)
$$

and

$$
\alpha_{n}(\mathbf{x})=n^{-1 / 2} \sum_{i=1}^{n}\left\{\prod_{k=1}^{m} \mathbf{1}\left(\varepsilon_{i+k-1} \leq x_{k}\right)-K(\mathbf{x})\right\},
$$

where inequalities between vectors hold componentwise.

Assumption I. $F$ admits a continuous derivative $F^{\prime}$ that is square-integrable.

Assumption II. There exist $\alpha, \beta_{1}, \ldots, \beta_{m}$ in the Skorohod space $\mathcal{D}\left([-\infty, \infty]^{m}\right)$ of càdlàg processes such that for each $k \in\{1, \ldots, m\}, \beta_{k}\left(x_{1}, \ldots, x_{m}\right)$ is not a function of $x_{k}$ and such that $\alpha_{n} \rightsquigarrow \alpha$ and $\mathbb{K}_{n}=n^{1 / 2}\left(K_{n}-K\right) \rightsquigarrow \mathbb{K}$ in $\mathcal{D}\left([-\infty, \infty]^{m}\right)$ as $n \rightarrow \infty$, where for all $\mathbf{x}=\left(x_{1}, \ldots, x_{m}\right) \in[-\infty, \infty]^{m}, \mathbb{K}(\mathbf{x})=$ $\alpha(\mathbf{x})-\sum_{k=1}^{m} F^{\prime}\left(x_{k}\right) \beta_{k}(\mathbf{x})$.

Theorem 1. Suppose that Assumptions I and II hold under $H_{0}$. If $\mathbb{K} \neq \alpha$, then further assume that $F$ is symmetric. Then for all $\delta \in(0, \infty), \tilde{S}_{n, \delta} \rightsquigarrow \mathcal{N}(0,1)$ as $n \rightarrow \infty$.

A distinct advantage of $\tilde{S}_{n, \delta}$ over $S_{n, \delta}$ is that when parameters need not be estimated, its finite-sample distribution does not depend on $F$. A reliable approximation thereof can be obtained through Algorithm 2. When working with residuals, both $S_{n, \delta}$ and $\tilde{S}_{n, \delta}$ have finite-sample distributions that depend on parameter estimates.

\section{EMPIRICAL PROCESSES EXTENDING $S_{n, \delta}$ AND $\tilde{S}_{n, \delta}$}

Although the rank-based statistic $\tilde{S}_{n, \delta}$ does not suffer from the slow rate of convergence associated with the original BDS statistic $S_{n, \delta}$, it still shares with it a dependence on the proximity parameter $\delta>0$. In this section we describe empirical processes that we exploit in Section 4 to get rid of this arbitrariness.

\subsection{An Empirical Process Extending $S_{n, \delta}$}

Consider the empirical process $B_{n}$ defined for each $\mathbf{t}=\left(t_{1}\right.$, $\left.\ldots, t_{m}\right) \in[0, \infty]^{m}$ by

$$
B_{n}(\mathbf{t})=\frac{1}{\left(\begin{array}{l}
n \\
2
\end{array}\right)} \sum_{1 \leq i<j \leq n} \prod_{k=1}^{m} \mathbf{1}\left(\left|w_{j k}-w_{i k}\right| \leq t_{k}\right)
$$

and let $G_{n}(\delta)=B_{n}(\delta, \infty, \ldots, \infty)$ for every $\delta \in[0, \infty]$. A test based on

$$
\mathbb{D}_{n}(\mathbf{t})=n^{1 / 2}\left\{B_{n}(\mathbf{t})-\prod_{k=1}^{m} G_{n}\left(t_{k}\right)\right\}
$$

would then provide an extension of $S_{n, \delta}$. For, $S_{m}(\delta)=B_{n}(\delta$, $\ldots, \delta), S(\delta)=G_{n}(\delta)$, and thus $S_{n, \delta}=\mathbb{D}_{n}(\delta, \ldots, \delta) / s_{n}(\delta)$. The limit of $\mathbb{D}_{n}$ is given in Theorem 2, along with that of the related processes $\mathbb{B}_{n}=n^{1 / 2}\left(B_{n}-B\right)$ and $\mathbb{B}_{n}^{\star}=2 n^{1 / 2}\left(B_{n}^{\star}-B\right)$, where

$$
\begin{aligned}
B(\mathbf{t}) & =\prod_{k=1}^{m} G\left(t_{k}\right), \\
B_{n}^{\star}(\mathbf{t}) & =n^{-1} \sum_{i=1}^{n} \prod_{k=1}^{m}\left\{F\left(w_{i k}+t_{k}\right)-F\left(w_{i k}-t_{k}\right)\right\},
\end{aligned}
$$

and, for all $\delta \in[0, \infty], G(\delta)=P\left(\left|\varepsilon_{2}-\varepsilon_{1}\right| \leq \delta\right)$. When $F$ is known (but only then), the processes $\mathbb{B}_{n}$ and $\mathbb{B}_{n}^{\star}$ could be used instead of $\mathbb{D}_{n}$ to build tests of randomness. 
Theorem 2. Under $H_{0}$ and Assumptions I and II, $\left(\mathbb{B}_{n}, \mathbb{B}_{n}^{\star}\right.$, $\left.\mathbb{D}_{n}\right) \rightsquigarrow(\mathbb{B}, \mathbb{B}, \mathbb{D})$ as $n \rightarrow \infty$ in $\mathcal{D}\left([0, \infty]^{m}\right)^{\otimes 3}$, where $\mathbb{B}$ and $\mathbb{D}$ are continuous centered Gaussian processes with covariances $\Gamma_{\mathbb{B}}$ and $\Gamma_{\mathbb{D}}$. Let $\gamma(\kappa, \lambda)=P\left(\left|\varepsilon_{2}-\varepsilon_{1}\right| \leq \kappa,\left|\varepsilon_{3}-\varepsilon_{1}\right| \leq \lambda\right)$ for all $\kappa, \lambda \in[0, \infty]$. Then for all $\mathbf{s}=\left(s_{1}, \ldots, s_{m}\right), \mathbf{t}=\left(t_{1}, \ldots, t_{m}\right) \in$ $[0, \infty)^{m}$

$$
\begin{aligned}
\Gamma_{\mathbb{B}}(\mathbf{s}, \mathbf{t})= & 4\left\{\prod_{k=1}^{m} \gamma\left(s_{k}, t_{k}\right)-\prod_{k=1}^{m} G\left(s_{k}\right) G\left(t_{k}\right)\right\} \\
& +4 \sum_{j=2}^{m}\left\{\prod_{k=1}^{j-1} G\left(s_{k}\right) G\left(t_{m+1-k}\right)\right\} \\
& \times\left\{\prod_{k=j}^{m} \gamma\left(s_{k}, t_{k+1-j}\right)-\prod_{k=j}^{m} G\left(s_{k}\right) G\left(t_{k+1-j}\right)\right\} \\
& +4 \sum_{j=2}^{m}\left\{\prod_{k=1}^{j-1} G\left(s_{m+1-k}\right) G\left(t_{k}\right)\right\} \\
& \times\left\{\prod_{k=j}^{m} \gamma\left(s_{k+1-j}, t_{k}\right)-\prod_{k=j}^{m} G\left(s_{k+1-j}\right) G\left(t_{k}\right)\right\},
\end{aligned}
$$

and

$$
\Gamma_{\mathbb{D}}(\mathbf{s}, \mathbf{t})=\Gamma_{\mathbb{B}}(\mathbf{s}, \mathbf{t})-4 B(\mathbf{s}) B(\mathbf{t}) \sum_{j=1}^{m} \sum_{k=1}^{m}\left\{\frac{\gamma\left(s_{j}, t_{k}\right)}{G\left(s_{j}\right) G\left(t_{k}\right)}-1\right\} .
$$

Theorem 2 implies that, as with the classical BDS statistic, the asymptotic covariances $\Gamma_{\mathbb{B}}$ and $\Gamma_{\mathbb{D}}$ do not depend on the model parameters or their estimates. However, they do depend on $F$ through $G$ and $\gamma$. From Proposition A.2 in Appendix A, consistent estimators of $G(\delta)$ and $\gamma(\kappa, \lambda)$ are given by $G_{n}(\delta)$ and

$$
\gamma_{n}(\kappa, \lambda)=n^{-3} \sum_{i=1}^{n} \sum_{j=1}^{n} \sum_{k=1}^{n} \mathbf{1}\left(\left|e_{i}-e_{k}\right| \leq \kappa\right) \mathbf{1}\left(\left|e_{j}-e_{k}\right| \leq \lambda\right) .
$$

\subsection{An Empirical Process Extending $\tilde{S}_{n, \delta}$}

Results parallel to those of Section 3.1 are available when the $e_{i}$ 's are replaced by the rank-based $\tilde{e}_{i}$ 's. Write $\tilde{\mathbf{w}}_{i}=$ $\left(\tilde{w}_{i 1}, \ldots, \tilde{w}_{i m}\right)=\left(\tilde{e}_{i}, \ldots, \tilde{e}_{i+m-1}\right)$ for $i \in\{1, \ldots, n\}$. For all $\mathbf{u}=\left(u_{1}, \ldots, u_{m}\right) \in[0,1]^{m}$, let

$$
\tilde{B}_{n}(\mathbf{u})=\frac{1}{\left(\begin{array}{c}
n \\
2
\end{array}\right)} \sum_{1 \leq i<j \leq n} \prod_{k=1}^{m} \mathbf{1}\left(\left|\tilde{w}_{j k}-\tilde{w}_{i k}\right| \leq u_{k}\right)
$$

and

$$
\tilde{B}_{n}^{\star}(\mathbf{u})=n^{-1} \sum_{i=1}^{n} \prod_{k=1}^{m}\left\{\tilde{F}\left(\tilde{w}_{i k}+u_{k}\right)-\tilde{F}\left(\tilde{w}_{i k}-u_{k}\right)\right\},
$$

where $\tilde{F}$ is the distribution function of a $\operatorname{Unif}(0,1)$. For every $\delta \in[0,1]$, also let

$$
\begin{aligned}
\tilde{G}_{n}(\delta) & =\tilde{B}_{n}(\delta, 1, \ldots, 1) \\
& =\frac{1}{\left(\begin{array}{l}
n \\
2
\end{array}\right)} \sum_{1 \leq i<j \leq n} \mathbf{1}\{|j-i| \leq(n+1) \delta\} \\
& =\frac{2 k}{n-1}-\frac{k(k+1)}{n(n-1)},
\end{aligned}
$$

whenever $k /(n+1) \leq \delta<(k+1) /(n+1)$ for some integer $k \in \mathbb{N}$.

Let $\tilde{G}$ be the distribution function of a $\operatorname{Beta}(1,2)$ and set $\tilde{B}(\mathbf{u})=\tilde{G}\left(u_{1}\right) \cdots \tilde{G}\left(u_{m}\right)$ for all $\mathbf{u}=\left(u_{1}, \ldots, u_{m}\right) \in[0,1]^{m}$. Analogs of $\mathbb{B}_{n}, \mathbb{B}_{n}^{\star}$, and $\mathbb{D}_{n}$ then may be defined by $\tilde{\mathbb{B}}_{n}=$ $n^{1 / 2}\left(\tilde{B}_{n}-\tilde{B}\right), \tilde{\mathbb{B}}_{n}^{\star}=2 n^{1 / 2}\left(\tilde{B}_{n}^{\star}-\tilde{B}\right)$, and

$$
\tilde{\mathbb{D}}_{n}(\mathbf{u})=n^{1 / 2}\left\{\tilde{B}_{n}(\mathbf{u})-\prod_{k=1}^{m} \tilde{G}_{n}\left(u_{k}\right)\right\} .
$$

Theorem 3. Suppose that Assumptions I and II hold under $H_{0}$. If $\mathbb{K} \neq \alpha$, then further assume that $F$ is symmetric. Then $\left(\tilde{\mathbb{B}}_{n}, \tilde{\mathbb{B}}_{n}^{\star}, \tilde{\mathbb{D}}_{n}\right) \rightsquigarrow(\tilde{\mathbb{B}}, \tilde{\mathbb{B}}, \tilde{\mathbb{B}})$ in $\mathcal{D}\left([0,1]^{m}\right)$ as $n \rightarrow \infty$, where $\tilde{\mathbb{B}}$ is a continuous centered Gaussian process with covariance $\Gamma_{\tilde{\mathbb{B}}}$ of the same form as $\Gamma_{\mathbb{D}}$ but with $G$ and $\gamma$ replaced by $\tilde{G}$ and $\tilde{\gamma}$. For all $\kappa, \lambda \in[0,1]$,

$$
\tilde{\gamma}(\kappa, \lambda)=\left\{\begin{array}{c}
-\kappa^{2} \lambda-2 \kappa \lambda^{2}+4 \kappa \lambda-\kappa^{3} / 3 \\
\text { when } \kappa \leq \min (\lambda, 1-\lambda) \\
\kappa-\lambda^{2}-\lambda \kappa^{2}+\left(\kappa^{3}-1\right) / 3-\kappa^{2}+\lambda+2 \kappa \lambda \\
\text { when } \kappa \geq \max (\lambda, 1-\lambda) \\
-\lambda^{2} \kappa-2 \lambda \kappa^{2}+4 \lambda \kappa-\lambda^{3} / 3 \\
\text { when } \lambda \leq \min (\kappa, 1-\kappa) \\
\lambda-\kappa^{2}-\kappa \lambda^{2}+\left(\lambda^{3}-1\right) / 3-\lambda^{2}+\kappa+2 \lambda \kappa \\
\text { when } \lambda \geq \max (\kappa, 1-\kappa) .
\end{array}\right.
$$

Note that under $H_{0}$, Theorem 3 entails $\tilde{S}_{n, \delta}=\tilde{\mathbb{D}}_{n}(\delta, \ldots, \delta) /$ $\tilde{s}_{n}(\delta) \rightsquigarrow \mathcal{N}(0,1)$ as $n \rightarrow \infty$ for any $\delta \in(0,1)$, where $\tilde{s}_{n}^{2}(\delta)=$ $s_{n}^{2}\left(\tilde{e}_{1}, \ldots, \tilde{e}_{n}\right) \rightarrow \Gamma_{\tilde{\mathbb{B}}}(\delta, \ldots, \delta)$. A similar comment applies to the analog statistic

$$
\tilde{S}_{n, \delta}^{\star}=\tilde{\mathbb{B}}_{n}^{\star}(\delta, \ldots, \delta) / \tilde{s}_{n}(\delta) .
$$

Remark 1. There is little difference between $\tilde{\mathbb{B}}_{n}$ and $\tilde{\mathbb{D}}_{n}$. In fact,

$\sup _{\mathbf{u} \in[0,1]^{m}}\left|\tilde{\mathbb{D}}_{n}(\mathbf{u})-\tilde{\mathbb{B}}_{n}(\mathbf{u})\right| \leq m \sup _{\delta \in[0,1]}\left|\tilde{G}_{n}(\delta)-\tilde{G}(\delta)\right|=O\left(n^{-1}\right)$,

which explains why they have the same limit. In the sequel, statistics based on $\tilde{\mathbb{D}}_{n}$ are preferred over those based on $\tilde{\mathbb{B}}_{n}$, because the former process is less biased and no extra computational cost is associated with the use of $\tilde{G}_{n}$.

\section{STATISTICS BASED ON FUNCTIONAL EXTENSIONS}

In view of Theorems 2 and 3, extensions of statistics $S_{n, \delta}$ and $\tilde{S}_{n, \delta}$ could be based on quadratic forms involving either $\mathbb{D}_{n}(\mathbf{t})$ or $\tilde{\mathbb{D}}_{n}(\mathbf{u})$ for finitely many vectors $\mathbf{t} \in[0, \infty)^{m}, \mathbf{u} \in[0,1)^{m}$. Once properly normalized, these quadratic forms would be asymptotically distributed as chi-squared random variables. This approach would provide no relief, however, because the quadratic form would depend on several arbitrary choices of $\mathbf{t}$ or $\mathbf{u}$ rather than on $\delta$. In addition, the issue related to the slow rate of convergence would remain for test statistics based on $\mathbb{D}_{n}$ or $\tilde{\mathbb{D}}_{n}$.

One obvious way around the arbitrariness of quadratic forms based on a finite number of evaluations of $\mathbb{D}_{n}$ or $\tilde{\mathbb{D}}_{n}$ is to resort to continuous functionals of these empirical processes that take into account their value over an infinite number of points. We consider several statistics of this type here. 


\subsection{The Case Where $F$ Is Known}

In the spirit of freeing the BDS statistic from $\delta$, an option would be to integrate $S_{n, \delta}$ over all values of this parameter. This idea leads naturally to $I_{n}=\int \mathbb{D}_{n}(\delta, \ldots, \delta) d G(\delta)$, which can be computed because $G$ is known through $F$. Another possible choice is $I_{n}^{\star}=\int \mathbb{B}_{n}^{\star}(\delta, \ldots, \delta) d G(\delta)$. By Theorem 2, $I_{n}$ and $I_{n}^{\star}$ converge in law to centered Gaussian variables with variances $\iint \Gamma_{\mathbb{D}}\left(\delta, \ldots, \delta, \delta^{\prime}, \ldots, \delta^{\prime}\right) d G(\delta) d G\left(\delta^{\prime}\right)$ and $\iint \Gamma_{\mathbb{B}}\left(\delta, \ldots, \delta, \delta^{\prime}, \ldots, \delta^{\prime}\right) d G(\delta) d G\left(\delta^{\prime}\right)$.

Other natural extensions of $S_{n, \delta}$ based on the empirical processes $\mathbb{D}_{n}$ and $\mathbb{B}_{n}^{\star}$ could be constructed from the Kolmogorov-Smirnov functional by setting

$$
\begin{aligned}
M_{n} & =\sup _{\delta \in[0, \infty)}\left|\mathbb{D}_{n}(\delta, \ldots, \delta)\right| \quad \text { and } \\
M_{n}^{\star} & =\sup _{\delta \in[0, \infty)}\left|\mathbb{B}_{n}^{\star}(\delta, \ldots, \delta)\right| .
\end{aligned}
$$

Under the conditions of Theorem 2, and as $n \rightarrow \infty$, these statistics converge weakly to sup $|\mathbb{D}(\delta, \ldots, \delta)|$ and $\sup |\mathbb{B}(\delta, \ldots, \delta)|$. The Cramér-von Mises functional is another option that leads to statistics $T_{n}=\int \mathbb{B}_{n}^{2}(\mathbf{t}) d B(\mathbf{t})$ and $T_{n}^{\star}=\int \mathbb{B}_{n}^{\star 2}(\mathbf{t}) d B(\mathbf{t})$. By Theorem 2, the asymptotic distributions of $T_{n}$ and $T_{n}^{\star}$ are $\int \mathbb{D}^{2}(\mathbf{t}) d B(\mathbf{t})$ and $\int \mathbb{B}^{2}(\mathbf{t}) d B(\mathbf{t})$, which are infinite sums of weighted chi-squared random variables.

In view of the slow speed of convergence of the statistics $S_{n, \delta}, I_{n}, I_{n}^{\star}, M_{n}, M_{n}^{\star}, T_{n}$, and $T_{n}^{\star}$ to limits that involve $F$ in an intricate way, it seems wiser to rely on their finite-sample distributions for testing purposes. The following algorithm is for the case of $T_{n}$. Its validity stems from Theorem 2. Modifications needed for other functionals of $\mathbb{D}_{n}, \mathbb{B}_{n}^{\star}$, or even $\mathbb{B}_{n}$ are obvious.

Algorithm 1. For each $\ell \in\{1, \ldots, L\}$, draw a random sample $E_{\ell}=\left\{e_{1}, \ldots, e_{n}\right\}$ from $F$, set $e_{i+n}=e_{i}$ for all $i \in \mathbb{N}$, and compute $T_{n, \ell}\left(E_{\ell}\right)$. Critical values of $T_{n}$ can be estimated by quantiles of $\left\{T_{n, 1}, \ldots, T_{n, L}\right\}$, whereas an approximate $p$ value for an observed statistic $T_{n, 0}$ is given by $\sum_{\ell=1}^{L} \mathbf{1}\left(T_{n, \ell}>T_{n, 0}\right) / L$ for large $L$.

\subsection{The Case Where $F$ Is Unknown}

When $F$ is unknown and either $\mathbb{K}=\alpha$ or $F$ is symmetric, rank-based analogs of $I_{n}$ and $I_{n}^{\star}$ are given by

$$
\begin{aligned}
\tilde{I}_{n}= & \int_{0}^{1} \tilde{\mathbb{D}}_{n}(\delta, \ldots, \delta) d \tilde{G}(\delta) \\
= & n^{1 / 2}\left[\frac{1}{\left(\begin{array}{l}
n \\
2
\end{array}\right)} \sum_{1 \leq i<j \leq n} \min _{1 \leq k \leq m}\left(1-\left|\tilde{w}_{i k}-\tilde{w}_{j k}\right|\right)^{2}\right. \\
& \left.-\sum_{k=0}^{n} \frac{2(n-k)+1}{(n+1)^{2}}\left\{\frac{2 n k-k(k+1)}{n(n-1)}\right\}^{m}\right]
\end{aligned}
$$

and

$$
\begin{aligned}
\tilde{I}_{n}^{\star} & =\int_{0}^{1} \tilde{\mathbb{B}}_{n}^{\star}(\delta, \ldots, \delta) d \tilde{G}(\delta) \\
& =2 n^{-1 / 2} \sum_{i=1}^{n}\left\{\tilde{W}_{m}\left(\tilde{\mathbf{w}}_{i}\right)-\frac{1}{m+1}\right\} .
\end{aligned}
$$

Here $\tilde{W}_{p}$ is symmetric in its arguments and can be expressed in the form

$$
\begin{aligned}
\tilde{W}_{p}(\mathbf{u})=2 \sum_{k=0}^{p} \int_{u_{k}}^{u_{k+1}}\left\{(1-\xi)(2 \xi)^{p-k}\right. & \prod_{j=1}^{k}\left(u_{j}+\xi\right) \\
& \left.+\xi \prod_{j=1}^{k}\left(u_{j}+1-\xi\right)\right\} d \xi
\end{aligned}
$$

for all $\mathbf{u}=\left(u_{1}, \ldots, u_{p}\right)$ such that $0=u_{0} \leq u_{1} \leq \cdots \leq u_{p} \leq$ $u_{p+1}=1 / 2$. In addition,

$$
\tilde{W}_{p}(\mathbf{u})=\tilde{W}_{p}\left\{u_{1} \wedge\left(1-u_{1}\right), \ldots, u_{p} \wedge\left(1-u_{p}\right)\right\},
$$

where $u_{j} \wedge\left(1-u_{j}\right)=\min \left(u_{j}, 1-u_{j}\right)$ for all $j \in\{1, \ldots, p\}$. By Theorem 3, both $\tilde{I}_{n}$ and $\tilde{I}_{n}^{\star}$ converge in law to the centered Gaussian variable $\tilde{I}=\int \tilde{\mathbb{B}}(\delta, \ldots, \delta) d \tilde{G}(\delta)$ with variance $\iint \Gamma_{\tilde{\mathbb{B}}}\left(\delta, \ldots, \delta, \delta^{\prime}, \ldots, \delta^{\prime}\right) d \tilde{G}(\delta) d \tilde{G}\left(\delta^{\prime}\right)$.

Rank-based analogs of $M_{n}$ and $M_{n}^{\star}$ are given by

$$
\tilde{M}_{n}=\max _{i \in\{1, \ldots, n\}}\left|\tilde{\mathbb{D}}_{n}\left(\frac{i}{n+1}, \ldots, \frac{i}{n+1}\right)\right|
$$

and

$$
\tilde{M}_{n}^{\star}=\max _{i \in\{1, \ldots, n\}}\left|\tilde{\mathbb{B}}_{n}^{\star}\left(\frac{i}{n+1}, \ldots, \frac{i}{n+1}\right)\right| .
$$

Under the conditions stated in Theorem 3, they converge weakly to the same limit $\sup |\tilde{\mathbb{B}}(\delta, \ldots, \delta)|$ as $n \rightarrow \infty$. Similarly, a rank-based version of $T_{n}$ is given by

$$
\begin{aligned}
\tilde{T}_{n}= & \int_{[0,1]^{m}} \tilde{\mathbb{D}}_{n}^{2}(\mathbf{u}) d \tilde{B}(\mathbf{u}) \\
= & \frac{4}{n(n-1)^{2}} \sum_{1 \leq i_{1}<j_{1} \leq n} \sum_{1 \leq i_{2}<j_{2} \leq n} \prod_{k=1}^{m}\{1 \\
& \left.-\tilde{G}\left(\left|w_{i_{1} k}-w_{j_{1} k}\right| \vee\left|w_{i_{2} k}-w_{j_{2} k}\right|\right)\right\} \\
& +n\left\{\frac{5 n^{3}+n^{2}-4}{15 n\left(n^{2}-1\right)}\right\}^{m} \\
& -\frac{4}{n-1} \sum_{1 \leq i<j \leq n} \prod_{k=1}^{m} N\left\{(n+1)\left|\tilde{w}_{i k}-\tilde{w}_{j k}\right|\right\},
\end{aligned}
$$

where, for arbitrary $x, y \in[0, \infty), x \vee y=\max (x, y)$ and

$$
\begin{aligned}
N(x)= & \left(-3 x^{4}+(12 n+4) x^{3}-\left(12 n^{2}+18 n-3\right) x^{2}\right. \\
& \left.+\left(12 n^{2}+6 n-4\right) x\right) /\left(6 n(n-1)(n+1)^{2}\right) \\
& +\frac{3 n+2}{6(n+1)} .
\end{aligned}
$$

Finally, a rank-based version of $T_{n}^{\star}$ is

$$
\begin{aligned}
\tilde{T}_{n}^{\star}= & \int_{[0,1]^{m}}\left|\tilde{\mathbb{B}}_{n}^{\star}(\mathbf{u})\right|^{2} d \tilde{B}(\mathbf{u}) \\
= & \frac{4 n}{3^{m}}+\frac{4}{n} \sum_{i=1}^{n} \sum_{j=1}^{n} \prod_{k=1}^{m} \tilde{W}_{2}\left(\tilde{w}_{i k}, \tilde{w}_{j k}\right) \\
& -8 \sum_{j=1}^{n} \prod_{k=1}^{m} \tilde{N}\left(\tilde{w}_{j k}\right) .
\end{aligned}
$$


In these formulas,

$$
\begin{aligned}
\tilde{W}_{2}\left(u^{\prime}, v^{\prime}\right)= & \tilde{W}_{2}(u, v) \\
= & \int_{0}^{1}\left\{\tilde{F}\left(u^{\prime}+s\right)-\tilde{F}\left(u^{\prime}-s\right)\right\} \\
& \times\left\{\tilde{F}\left(v^{\prime}+s\right)-\tilde{F}\left(v^{\prime}-s\right)\right\} d \tilde{G}(s) \\
= & 1 / 6+u v(1-u \vee v)+u\left(1+u^{3}\right) / 3 \\
& +v\left(1+v^{3}\right) / 3-(u \wedge v)^{3}-2(u \vee v)^{3} / 3,
\end{aligned}
$$

with $u=u^{\prime} \wedge\left(1-u^{\prime}\right), v=v^{\prime} \wedge\left(1-v^{\prime}\right)$, and $\tilde{N}\left(u^{\prime}\right)=\tilde{N}(u)$, where

$$
\begin{aligned}
\tilde{N}(u) & =\int_{0}^{1}\{\tilde{F}(u+s)-\tilde{F}(u-s)\} \tilde{G}(s) d \tilde{G}(s) \\
& =187 / 480-3(u-1 / 2)^{2} / 4+(u-1 / 2)^{4} / 2 .
\end{aligned}
$$

The following algorithm, stated here for the statistic $\left|\tilde{S}_{n, \delta}\right|$, can be used mutatis mutandis to approximate the null distribution of $\tilde{I}_{n}, \tilde{M}_{n}, \tilde{T}_{n}, \tilde{S}_{n, \delta}^{\star}, \tilde{I}_{n}^{\star}, \tilde{M}_{n}^{\star}, \tilde{T}_{n}^{\star}$, or other functionals of $\tilde{\mathbb{D}}_{n}$, $\tilde{\mathbb{B}}_{n}^{\star}$, or even $\tilde{\mathbb{B}}_{n}$. Its validity stems from Theorem 3 .

Algorithm 2. Draw random permutations $\pi_{1}, \ldots, \pi_{L}$ of $\{1, \ldots, n\}$. Compute $\tilde{S}_{n, \delta, \ell}=S_{n, \delta}\left(\tilde{e}_{1}, \ldots, \tilde{e}_{n+m-1}\right)$, where $\tilde{e}_{i+n}=\tilde{e}_{i}=\pi_{\ell}(i) /(n+1)$ for $i \in\{1, \ldots, n\}$. Critical values of $\tilde{S}_{n, \delta}$ can be estimated by quantiles of $\left\{\tilde{S}_{n, \delta, 1}, \ldots, \tilde{S}_{n, \delta, L}\right\}$, whereas an approximate $p$ value for an observed $\left|\tilde{S}_{n, \delta, 0}\right|$ is given by $\sum_{\ell=1}^{L} \mathbf{1}\left(\left|\tilde{S}_{n, \delta, \ell}\right|>\left|\tilde{S}_{n, \delta, 0}\right|\right) / L$ for large $L$.

\section{FINITE-SAMPLE PERFORMANCE}

This section presents three sets of Monte Carlo experiments comparing the performance, for a sample size $n=100$, under various alternatives and choices of dimension $m$, of the statistics (1)-(9). To estimate the power under a fixed alternative, 10,000 samples were generated for each statistic, and the percentage of rejected samples was recorded. To speed up calculations, the 95\% quantiles of Table 1 were used instead of $p$ values.

In the simulations, $\delta=.3$ was used for $\tilde{S}_{n, \delta}$ and $\tilde{S}_{n, \delta}^{\star}$. Given that the standard deviation of $\tilde{e}_{i}$ is $1 / 12^{1 / 2} \approx .3$, this choice corresponds to the midpoint of the interval $[\hat{\sigma} / 2,3 \hat{\sigma} / 2]$, where $\hat{\sigma}$ is the estimated standard deviation of the innovations, following the recommendation of Brock et al. (1996).

Standard Gaussian variates were assumed in computing the quantiles of $S_{n, \hat{\sigma}}$. Accordingly, this statistic maintains its level.
Although this decision allows for meaningful power comparisons, it confers an undue advantage to the BDS statistic. In applications, practitioners would rely on a critical value of 1.96 , because the statistic has a standard Gaussian distribution asymptotically. But this would result in an (often highly) inappropriate type I error, as illustrated in Table 6 (Sec. 5.2). Consequently, the power of the classical BDS statistic is often unrealistic unless $F$ is known. For this reason, its performance is not explicitly discussed in the sequel.

In the first two series of experiments, reported in Sections 5.1 and 5.2, model parameters are assumed known. Because innovations are then observed, $\mathbb{K}=\alpha$ and the proposed methodology is valid whether or not $F$ is symmetric. In the final experiment, presented in Section 5.3, data generated from an AR(2) model are fitted using an AR(1) model, and tests of randomness are performed on the residuals. The three sets of results are discussed in Section 5.4, and a theoretical argument explaining the difference between statistics based on $\tilde{\mathbb{D}}_{n}$ and $\tilde{\mathbb{B}}_{n}^{\star}$ is given in Section 5.5.

\subsection{First Experiment}

Comparisons were first made for nine models of the form $\varepsilon_{i}=\varphi\left(\varepsilon_{i-1}, u_{i}, u_{i-1}\right)$ listed in Table 2. Following Hong and White (2005), the white noise $u_{i}$ was taken to be Gaussian, and their procedures were followed to obtain nearly stationary time series. For each repetition and for each alternative, a time series of length 200 was generated, with only the final 100 observations used.

For models A1-A9, Table 3 gives the estimated power of statistics (1)-(9) with $m=2$. The corresponding results for the statistic $\mathcal{T}_{n}(1)$ of Hong and White (2005) were kindly provided by these authors. Table 4 presents similar results for dimensions $m \in\{2,4,6\}$, for all statistics but $\mathcal{T}_{n}(1)$.

\subsection{Second Experiment}

Additional comparisons were made using models B1-B9 listed in Table 5 in which a parameter $\theta$ governs dependence and $\theta=0$ corresponds to independence. Model B7 is due to

\begin{tabular}{|c|c|c|c|c|c|c|}
\hline$S_{n, \hat{\sigma}}$ & (1) & 2.407289 & 2.437270 & 2.515284 & 2.561355 & 2.675926 \\
\hline$\tilde{S}_{n, .3}$ & (2) & 3.751936 & 4.024279 & 4.521843 & 5.042841 & 5.655694 \\
\hline$\tilde{S}_{n, .3}^{\star}$ & (3) & 3.677803 & 2.945949 & 2.639806 & 2.458066 & 2.327052 \\
\hline$\tilde{I}_{n}$ & (4) & .038934 & .055301 & .064259 & .069244 & .071085 \\
\hline$\tilde{M}_{n}^{\star}$ & (7) & .141433 & .209432 & .259929 & .300341 & .335972 \\
\hline$\tilde{T}_{n}$ & (8) & .001865 & .002670 & .002234 & .001536 & .000923 \\
\hline$\tilde{T}_{n}^{\star}$ & (9) & .004048 & .004104 & .002972 & .001784 & .000956 \\
\hline
\end{tabular}
Tong and Lim (1980). For model B8 of Genest, Quessy, and Rémillard (2002), $\theta=1 / 4$ corresponds to the deterministic tent map, for which traditional measures of dependence for the pairs $\left(X_{i}, X_{i+\ell}\right)$ vanish. As was shown by Chatterjee and Yilmaz (1992), the tent map exemplifies chaotic behavior.

Table 1. The 95\% quantiles for statistics (1)-(9) for $n=100$ and for $m \in\{2,3,4,5,6\}$, based on 10,000 replicates 
Table 2. Models with Gaussian noise $u_{i}$

\begin{tabular}{lll}
\hline \hline Model & Name & Equation \\
\hline A1 & iid & $\varepsilon_{i}=u_{i}$ \\
A2 & AR(1) & $\varepsilon_{i}=.3 \varepsilon_{i-1}+u_{i}$ \\
A3 & ARCH(1) & $\varepsilon_{i}=h_{i}^{1 / 2} u_{i}, h_{i}=1+.8 \varepsilon_{i-1}^{2}$ \\
A4 & Threshold GARCH(1,1) & $\varepsilon_{i}=h_{i}^{1 / 2} u_{i}$, with $h_{i}^{2}=.25+.6 h_{i-1}^{2}+.5 \varepsilon_{i-1}^{2} \mathbf{1}\left(u_{i-1}<0\right)+.2 \varepsilon_{i-1}^{2} \mathbf{1}\left(u_{i-1} \geq 0\right)$ \\
A5 & Bilinear AR(1) & $\varepsilon_{i}=.8 \varepsilon_{i-1} u_{i-1}+u_{i}$ \\
A6 & Nonlinear MA(1) & $\varepsilon_{i}=.8 u_{i-1}^{2}+u_{i}$ \\
A7 & Threshold AR(1) & $\varepsilon_{i}=.4 \varepsilon_{i-1} \mathbf{1}\left(\varepsilon_{i-1}>1\right)-.5 \varepsilon_{i-1} \mathbf{1}\left(\varepsilon_{i-1} \leq 1\right)+u_{i}$ \\
A8 & Fractional AR(1) & $\varepsilon_{i}=.8\left|\varepsilon_{i-1}\right|^{1 / 2}+u_{i}$ \\
A9 & Sign AR(1) & $\varepsilon_{i}=\operatorname{sign}\left(\varepsilon_{i-1}\right)+.43 u_{i}$ \\
\hline
\end{tabular}

Table 6 gives the rejection rates for samples of size $n=100$ for the test based on $\tilde{S}_{n, 3}$ when $m \in\{2,4,6\}$ under the 27 alternatives corresponding to models B1-B9 with $\theta \in\{0,1 / 8,1 / 4\}$. It also reports the rejection rates for the test based on $S_{n, \hat{\sigma}}$ with critical point 1.96 . As shown, the latter statistic generally fails to maintain its level and would be preferable to $\tilde{S}_{n, .3}$ only for models B5 and B6.

Finally, Table 7 compares the performance of statistics (1)(9) when $m \in\{2,4,6\}$ and $\theta=1 / 4$ in models B1-B9. To achieve stationarity for a given times series model, 200 observations were generated for each replicate, with only the final 100 used.

\subsection{Third Experiment}

For the last set of comparisons, data were generated from an AR(2) model,

$$
x_{i}=-2+.3 x_{i-1}+\epsilon_{i}, \quad \epsilon_{i}=\theta \epsilon_{i-1}+u_{i},
$$

with standard Gaussian white noise innovation $u_{i}$ for every integer $i \geq 1$. Equivalently, we could write $x_{i}-(\theta+.3) x_{i-1}+$ $.3 \theta x_{i-2}=2(\theta-1)+u_{i}$. However, an AR(1) model then would be assumed for $x_{i}$; this would be wrong unless $\theta=0$.

Table 8 reports the performance of the statistics (1)-(9) for embedding dimensions $m \in\{2,3,4,5,6\}$ and two alternatives corresponding to $\theta \in\{0, .8\}$. As in earlier experiments, near stationarity was obtained by generating 200 observations of each time series and then dropping the first 100 of these.

\subsection{Discussion}

First, as shown by the results given in Table 3, tests based on statistics $\tilde{S}_{n, 3}, \tilde{I}_{n}, \tilde{M}_{n}$, and $\tilde{T}_{n}$ have better power than the test based on $\mathcal{T}_{n}(1)$ for all models listed in Table 2 with the exception of A9, where they come very close. As for the tests based on statistics $\tilde{S}_{n, 3}^{\star}, \tilde{I}_{n}^{\star}, \tilde{M}_{n}^{\star}$, and $\tilde{T}_{n}^{\star}$, they clearly dominate $\mathcal{T}_{n}(1)$ for models A3-A6. They are also comparable to $\mathcal{T}_{n}(1)$ for model A2 but are outperformed by it for models A7-A9.

Second, based on the results of Tables 3, 4, and 7, note that among the statistics derived from $\tilde{\mathbb{D}}_{n}$, the tests based on $\tilde{I}_{n}$ and $\tilde{T}_{n}$ are almost always optimal. But because of its computational complexity, $\tilde{T}_{n}$ is not so attractive. Among the statistics derived from $\tilde{\mathbb{B}}_{n}^{\star}, \tilde{I}_{n}^{\star}$ and $\tilde{T}_{n}^{\star}$ are the top performers, with $\tilde{S}_{n, 3}^{\star}$ not too far behind.

Third, there appears to be a significant difference between the performance of test statistics derived from $\tilde{\mathbb{D}}_{n}$ versus those based on $\tilde{\mathbb{B}}_{n}^{\star}$, depending on the class of alternatives. For most models with constant conditional variance given the past (e.g., B1-B4), the tests based on $\tilde{S}_{n, 3}, \tilde{I}_{n}, \tilde{M}_{n}$, or $\tilde{T}_{n}$ generally perform much better than those based on $\tilde{S}_{n, 3}^{\star}, \tilde{I}_{n}^{\star}, \tilde{M}_{n}^{\star}$, or $\tilde{T}_{n}^{\star}$. The opposite occurs for alternatives with nonconstant conditional variance given the past (e.g., B5-B6 and A4).

In practice, of course, the nature of the alternative is rarely known. For the $\mathcal{T}_{n}(1)$ statistic of Hong and White (2005), this is not a concern, because it tends to perform equally well whether or not the conditional variance is constant. But for the statistics proposed here, this might be problematic. Fortunately, the following general strategy can be used to circumvent the problem.

Table 3. Percentage of rejection of series of length $n=100$ of alternatives A1-A9 for tests at the 5\% level based on the statistics (1)-(9) and $\mathcal{T}_{n}(1)$ of Hong and White (2005)

\begin{tabular}{|c|c|c|c|c|c|c|c|c|c|c|}
\hline Model & $S_{n, \hat{\sigma}}$ & $\tilde{S}_{n, .3}^{\star}$ & $\tilde{I}_{n}^{\star}$ & $\tilde{M}_{n}^{\star}$ & $\tilde{T}_{n}^{\star}$ & $\tilde{S}_{n, .3}$ & $\tilde{I}_{n}$ & $\tilde{M}_{n}$ & $\tilde{T}_{n}$ & $\mathcal{T}_{n}(1)$ \\
\hline A1 & 5.12 & 4.62 & 5.22 & 4.79 & 5.12 & 5.29 & 5.02 & 5.21 & 5.01 & 6.5 \\
\hline A2 & 26.02 & 14.01 & 14.50 & 12.12 & 14.53 & 46.44 & 53.08 & 46.17 & 53.32 & 14.0 \\
\hline A4 & 65.07 & 72.13 & 72.35 & 65.86 & 72.96 & 44.61 & 57.74 & 49.60 & 54.39 & 20.6 \\
\hline A5 & 99.56 & 95.17 & 94.44 & 89.42 & 94.88 & 98.01 & 99.31 & 97.75 & 99.22 & 69.6 \\
\hline A6 & 78.32 & 51.50 & 50.03 & 39.46 & 50.92 & 67.30 & 70.05 & 61.27 & 76.24 & 34.0 \\
\hline A8 & 19.01 & 7.27 & 8.39 & 8.02 & 8.47 & 40.65 & 41.65 & 37.46 & 43.53 & 17.0 \\
\hline A9 & 61.59 & 31.85 & 34.10 & 32.58 & 33.97 & 57.98 & 59.97 & 58.77 & 59.17 & 60.8 \\
\hline
\end{tabular}

NOTE: Except for $\mathcal{T}_{n}(1)$, the percentage of rejection was estimated from 10,000 replicates. 
Table 4. Percentage of rejection of alternatives A1-A9 for tests at the 5\% level based on the statistics (1)-(9) with $m \in\{2,4,6\}$, as estimated from 10,000 replicates of series of length $n=100$

\begin{tabular}{|c|c|c|c|c|c|c|c|c|c|c|}
\hline Model & $m$ & $S_{n, \hat{\sigma}}$ & $\tilde{S}_{n, .3}^{\star}$ & $\tilde{I}_{n}^{\star}$ & $\tilde{M}_{n}^{\star}$ & $\tilde{T}_{n}^{\star}$ & $\tilde{S}_{n, .3}$ & $\tilde{I}_{n}$ & $\tilde{M}_{n}$ & $\tilde{T}_{n}$ \\
\hline \multirow[t]{2}{*}{ A1 } & 2 & 5.12 & 4.62 & 5.22 & 4.79 & 5.12 & 5.29 & 5.02 & 5.21 & 5.01 \\
\hline & 4 & 4.71 & 5.10 & 4.89 & 4.77 & 4.95 & 5.08 & 5.23 & 4.99 & 5.02 \\
\hline \multirow[t]{2}{*}{ A2 } & 2 & 26.02 & 14.01 & 14.50 & 12.12 & 14.53 & 46.44 & 53.08 & 46.17 & 53.32 \\
\hline & 4 & 19.04 & 12.23 & 11.29 & 10.85 & 12.07 & 33.05 & 38.09 & 32.10 & 45.14 \\
\hline \multirow{2}{*}{ A3 } & 4 & 85.83 & 91.60 & 90.48 & 89.43 & 91.85 & 64.82 & 84.01 & 80.01 & 84.14 \\
\hline & 6 & 75.87 & 85.82 & 84.05 & 84.39 & 85.95 & 49.04 & 76.88 & 74.00 & 77.05 \\
\hline \multirow{3}{*}{ A4 } & 2 & 65.07 & 72.13 & 72.35 & 65.86 & 72.96 & 44.61 & 57.74 & 49.60 & 54.39 \\
\hline & 4 & 77.51 & 84.25 & 82.02 & 80.93 & 83.61 & 55.49 & 73.50 & 68.87 & 73.07 \\
\hline & 6 & 78.09 & 86.15 & 83.94 & 84.56 & 85.93 & 53.77 & 76.98 & 74.38 & 77.16 \\
\hline \multirow{2}{*}{ A6 } & 4 & 60.00 & 36.76 & 32.73 & 30.68 & 35.19 & 50.85 & 33.28 & 22.01 & 58.55 \\
\hline & 6 & 42.95 & 29.02 & 25.97 & 26.28 & 28.43 & 32.57 & 18.58 & 13.40 & 41.72 \\
\hline \multirow[t]{3}{*}{ A7 } & 2 & 31.04 & 8.46 & 9.14 & 7.34 & 9.24 & 52.06 & 44.69 & 46.95 & 59.72 \\
\hline & 4 & 22.84 & 9.24 & 8.46 & 7.76 & 9.09 & 35.80 & 14.29 & 12.62 & 37.27 \\
\hline & 6 & 16.91 & 8.83 & 7.87 & 7.97 & 8.89 & 22.99 & 8.07 & 9.53 & 24.56 \\
\hline \multirow[t]{3}{*}{ A8 } & 2 & 19.01 & 7.27 & 8.39 & 8.02 & 8.47 & 40.65 & 41.65 & 37.46 & 43.53 \\
\hline & 4 & 14.14 & 7.12 & 7.60 & 7.43 & 7.65 & 27.53 & 24.64 & 19.75 & 32.31 \\
\hline & 6 & 11.98 & 6.97 & 6.90 & 6.69 & 7.34 & 18.89 & 15.16 & 12.47 & 26.06 \\
\hline \multirow[t]{2}{*}{ A9 } & 2 & 61.59 & 31.85 & 34.10 & 32.58 & 33.97 & 57.98 & 59.97 & 58.77 & 59.17 \\
\hline & 4 & 60.20 & 37.11 & 40.18 & 40.73 & 40.12 & 57.96 & 60.00 & 58.93 & 59.95 \\
\hline
\end{tabular}

Consider a statistic $\zeta\left(\tilde{\mathbb{D}}_{n}\right)$, computed from a continuous functional, $\zeta$, of $\tilde{\mathbb{D}}_{n}$ and its parent statistic $\zeta\left(\tilde{\mathbb{B}}_{n}^{\star}\right)$. Let $P_{n}$ and $P_{n}^{\star}$ represent the (approximate) $p$ values of $\zeta\left(\tilde{\mathbb{D}}_{n}\right)$ and $\zeta\left(\tilde{\mathbb{B}}_{n}^{\star}\right)$, as calculated using a method analogous to Algorithm 2 for $\tilde{T}_{n}$. A combined test of approximate level $\alpha^{*}$ is then obtained as follows: Reject $H_{0}$ if and only if $\min \left(P_{n}, P_{n}^{\star}\right)<\alpha^{*}$. To see that the limiting level of this decision rule is $\alpha^{*}$, note that under $H_{0}$, both statistics converge in law to the same random variable, $\zeta(\mathbb{B})$. Accordingly, both $P_{n}$ and $P_{n}^{\star}$ converge to the same uniform random variable on the interval $(0,1)$; thus $P\left(P_{n} \wedge P_{n}^{\star}<\alpha^{*}\right) \rightarrow \alpha^{*}$ as $n \rightarrow \infty$.

Finally, Table 8 portrays an instance of constant volatility. Based on the previous discussion, tests derived from $\tilde{\mathbb{D}}_{n}$ should deliver the best power. Indeed they do, despite their slight underestimation of the type I error when $m=2$ or 3 . It is particularly gratifying to see that $\tilde{I}_{n}$ and $\tilde{T}_{n}$ outperform the classical BDS statistic, even though the latter is used here under its most favorable conditions.

Table 5. Models used for the second experiment

\begin{tabular}{lll}
\hline \hline Number & Model & Equation \\
\hline B1 & AR(1) Gaussian & $\varepsilon_{i}=\theta \varepsilon_{i-1}+u_{i}$, with Gaussian noise $u_{i}$ \\
B2 & AR(1) Laplace & $\varepsilon_{i}=\theta \varepsilon_{i-1}+u_{i}$, with Laplace noise $u_{i}$ \\
B3 & AR(1) Cauchy & $\varepsilon_{i}=\theta \varepsilon_{i-1}+u_{i}$, with Cauchy noise $u_{i}$ \\
B4 & $\varepsilon_{i}=u_{i}-\theta u_{i-1}$, with Gaussian noise $u_{i}$ \\
B5 & MA(1) & $\varepsilon_{i}=h_{i}^{1 / 2} u_{i}$, with $h_{i}^{2}=1+\theta h_{i-1}^{2}+2 \theta \varepsilon_{i-1}^{2}$ \\
B6 & GARCH(1,1) & $\varepsilon_{i}=h_{i}^{1 / 2} u_{i}$, with $h_{i}=1+\theta \varepsilon_{i-1}^{2}$ \\
B7 & ARCH(1) & $\varepsilon_{i}=-\theta \varepsilon_{i-1} \operatorname{sign}\left(\varepsilon_{i-1}-.5\right)+u_{i}$, with noise $u_{i} \sim$ Unif(0, 1) \\
B8 & Threshold AR(1) & $\varepsilon_{i}=\left(1-\eta_{i}\right) u_{i}+\eta_{i}\left(1-\left|2 \varepsilon_{i-1}-1\right|\right)$, with iid $u_{i} \sim$ Unif $(0,1)$, independent \\
& (Randomized) & of the iid $\eta_{i} \sim \operatorname{Bernoulli}(4 \theta)$ \\
B9 & Tent map & $\varepsilon_{i}$ is Markovian, with $\left(\varepsilon_{i-1}, \varepsilon_{i}\right) \sim C_{\theta}$, with $C_{\theta}(u, v)=\left(u^{-\theta}+v^{-\theta}-1\right)^{-1 / \theta}$ \\
& Clayton copula & for $u, v \in(0,1)$ \\
\hline
\end{tabular}


Table 6. Percentage of rejection of alternatives B1-B9 with $\theta \in\{0,1 / 8,1 / 4\}$ for tests at the $5 \%$ level based on statistics $\tilde{S}_{n, .3}$ and $S_{n, \hat{\sigma}}$, as estimated from 10,000 replicates of series of length $n=100$

\begin{tabular}{|c|c|c|c|c|c|c|c|}
\hline \multirow[b]{2}{*}{ Model } & \multirow[b]{2}{*}{$m$} & \multicolumn{3}{|c|}{$\tilde{S}_{n, 3}$} & \multicolumn{3}{|c|}{$S_{n, \hat{\sigma}}$} \\
\hline & & $\theta=0$ & $\theta=1 / 8$ & $\theta=1 / 4$ & $\theta=0$ & $\theta=1 / 8$ & $\theta=1 / 4$ \\
\hline \multirow[t]{3}{*}{ B1 } & 2 & 5.27 & 9.97 & 31.55 & 4.45 & 6.17 & 15.99 \\
\hline & 4 & 4.81 & 7.84 & 21.38 & 4.80 & 5.60 & 12.41 \\
\hline & 6 & 5.15 & 6.77 & 14.65 & 5.65 & 5.67 & 10.51 \\
\hline \multirow[t]{3}{*}{ B2 } & 2 & 5.62 & 3.53 & 50.20 & 2.54 & 4.40 & 18.60 \\
\hline & 4 & 5.03 & 9.52 & 34.50 & 1.92 & 3.16 & 12.61 \\
\hline & 6 & 5.03 & 7.96 & 23.77 & 1.59 & 2.71 & 9.29 \\
\hline \multirow[t]{3}{*}{ B3 } & 2 & 4.67 & 52.30 & 95.12 & 4.63 & 30.03 & 55.96 \\
\hline & 4 & 4.89 & 35.23 & 86.53 & 4.49 & 21.88 & 41.52 \\
\hline & 6 & 5.11 & 23.93 & 71.67 & 3.54 & 18.02 & 28.68 \\
\hline \multirow[t]{3}{*}{ B4 } & 2 & 5.61 & 11.94 & 33.99 & 4.78 & 6.44 & 15.84 \\
\hline & 4 & 5.22 & 8.42 & 23.31 & 4.78 & 6.11 & 11.17 \\
\hline & 6 & 5.24 & 7.15 & 15.34 & 4.95 & 6.28 & 9.66 \\
\hline \multirow[t]{3}{*}{ B5 } & 2 & 5.58 & 18.85 & 51.40 & 4.99 & 34.69 & 75.19 \\
\hline & 4 & 5.19 & 15.09 & 50.84 & 4.91 & 28.84 & 76.31 \\
\hline & 6 & 5.05 & 11.67 & 40.27 & 5.17 & 23.52 & 69.23 \\
\hline \multirow[t]{3}{*}{ B6 } & 2 & 5.10 & 9.39 & 17.99 & 4.83 & 13.49 & 34.01 \\
\hline & 4 & 4.75 & 7.40 & 13.72 & 4.98 & 10.60 & 25.36 \\
\hline & 6 & 4.85 & 6.52 & 10.19 & 5.57 & 9.73 & 19.94 \\
\hline \multirow[t]{3}{*}{ B7 } & 2 & 5.64 & 88.87 & 100.00 & 22.49 & 93.29 & 100.00 \\
\hline & 4 & 4.99 & 73.20 & 100.00 & 27.94 & 85.40 & 100.00 \\
\hline & 6 & 5.23 & 52.10 & 100.00 & 34.86 & 75.87 & 100.00 \\
\hline \multirow[t]{3}{*}{ B8 } & 2 & 5.34 & 17.40 & 57.00 & 22.01 & 28.06 & 50.59 \\
\hline & 4 & 4.73 & 12.32 & 41.40 & 28.30 & 28.43 & 41.07 \\
\hline & 6 & 4.92 & 8.88 & 27.22 & 34.54 & 31.41 & 35.10 \\
\hline \multirow[t]{3}{*}{ B9 } & 2 & 5.36 & 7.88 & 19.98 & 22.48 & 25.78 & 36.94 \\
\hline & 4 & 5.11 & 6.89 & 13.70 & 27.59 & 30.05 & 36.05 \\
\hline & 6 & 4.68 & 5.78 & 10.32 & 34.80 & 36.22 & 38.16 \\
\hline
\end{tabular}

\subsection{Comparison Between Statistics Based on $\tilde{\mathbb{D}}_{n}$ and $\tilde{\mathbb{B}}_{n}^{\star}$}

By Theorem 3, statistics derived from $\tilde{\mathbb{D}}_{n}$ and $\tilde{\mathbb{B}}_{n}^{\star}$ have the same asymptotic behavior under $H_{0}$. However, under an alternative making the $\varepsilon_{i}$ 's dependent but stationary and ergodic with common continuous distribution $F$, their power should depend on $n^{1 / 2} \mu(\mathbf{w})$ and $n^{1 / 2} \mu^{\star}(\mathbf{w})$, where for every $\mathbf{w}=$ $\left(w_{1}, \ldots, w_{m}\right) \in[0,1]^{m}$,

$$
\begin{aligned}
\mu(\mathbf{w}) & =\tilde{B}(\mathbf{w})-\prod_{k=1}^{m} G\left(w_{k}\right) \quad \text { and } \\
\mu^{\star}(\mathbf{w}) & =\tilde{B}^{\star}(\mathbf{w})-2 \prod_{k=1}^{m} G\left(w_{k}\right),
\end{aligned}
$$

with

$$
\begin{gathered}
\tilde{B}(\mathbf{w})=P\left(\bigcap_{k=1}^{m}\left\{\left|U_{k}-V_{k}\right| \leq w_{k}\right\}\right) \quad \text { and } \\
\tilde{B}^{\star}(\mathbf{w})=2 P\left(\bigcap_{k=1}^{m}\left\{\left|U_{k}-W_{k}\right| \leq w_{k}\right\}\right)
\end{gathered}
$$

defined in terms of three independent random vectors $\mathbf{U}=$ $\left(U_{1}, \ldots, U_{m}\right), \mathbf{V}=\left(V_{1}, \ldots, V_{m}\right)$, and $\mathbf{W}=\left(W_{1}, \ldots, W_{m}\right)$ with uniform margins. Here $\mathbf{U}$ and $\mathbf{V}$ are independent observations from the copula $C$ associated with the random vector $\left(\varepsilon_{1}, \ldots, \varepsilon_{m}\right)$. As for $\mathbf{W}$, its elements are taken to be mutually independent.

Under the assumptions of stationarity and ergodicity, $\tilde{B}_{n}$ and $\tilde{B}_{n}^{\star}$ are convergent estimators of $\tilde{B}$ and $\tilde{B}^{\star}$. If $\mu \neq \equiv 0$, then the power of the test based on $\tilde{T}_{n}$ thus will tend to 1 as $n \rightarrow \infty$. In contrast, the standard BDS statistic and all tests based on $\tilde{B}_{n}(\delta, \ldots, \delta)$ are inconsistent whenever $\mu(\delta, \ldots, \delta)=0$ for all $\delta>0$, which is more frequent but still uncommon. Unless it occurs, simulations can be used to find the value of $\delta$ that maximizes $|\mu(\delta, \ldots, \delta)|=\left|\tilde{B}(\delta, \ldots, \delta)-\left(2 \delta-\delta^{2}\right)^{m}\right|$. From experience, it turns out that for many alternatives in the second list, $\delta=.3$ is close to optimum.

\section{ILLUSTRATIVE EXAMPLES}

To illustrate the use of the new procedures, the white noise hypothesis was tested using the logarithmic returns of the Standard \& Poor 500 index for the 251 trading days of the year 2006. This assumption is the strongest version of the market efficiency hypothesis, which is of economic relevance. The $p$ values for statistics (2)-(9) were estimated from 10,000 replicates. As in the simulations, $\delta=.3$ was used for $S_{n, \hat{\sigma}}$ and $\tilde{S}_{n, \delta}^{*}$. The null hypothesis could not be rejected at the $5 \%$ level for 
Table 7. Percentage of rejection of alternatives B1-B9 with $\theta=1 / 4$ for tests at the $5 \%$ level based on statistics (1)-(9), as estimated from 10,000 replicates of series of length $n=100$

\begin{tabular}{|c|c|c|c|c|c|c|c|c|c|c|}
\hline Model & $m$ & $S_{n, \hat{\sigma}}$ & $\tilde{S}_{n, .3}^{\star}$ & $\tilde{I}_{n}^{\star}$ & $\tilde{M}_{n}^{\star}$ & $\tilde{T}_{n}^{\star}$ & $\tilde{S}_{n, .3}$ & $\tilde{I}_{n}$ & $\tilde{M}_{n}$ & $\tilde{T}_{n}$ \\
\hline \multirow[t]{2}{*}{ B1 } & 2 & 15.99 & 10.00 & 10.77 & 9.37 & 10.85 & 31.55 & 36.45 & 31.39 & 37.35 \\
\hline & 4 & 12.41 & 9.50 & 9.46 & 9.09 & 9.79 & 21.38 & 24.46 & 20.05 & 30.14 \\
\hline \multirow[t]{2}{*}{ B2 } & 2 & 18.60 & 28.29 & 25.81 & 19.21 & 26.59 & 50.20 & 61.34 & 52.17 & 63.11 \\
\hline & 4 & 12.61 & 22.87 & 18.85 & 17.85 & 20.61 & 34.50 & 44.99 & 38.78 & 53.38 \\
\hline \multirow{2}{*}{ B3 } & 4 & 41.52 & 71.26 & 70.00 & 68.09 & 72.36 & 86.53 & 92.77 & 88.15 & 95.38 \\
\hline & 6 & 28.68 & 59.94 & 58.05 & 58.14 & 61.08 & 71.67 & 84.51 & 78.37 & 91.31 \\
\hline \multirow[t]{3}{*}{ B4 } & 2 & 15.84 & 10.82 & 10.85 & 8.90 & 11.10 & 33.99 & 38.78 & 33.14 & 39.28 \\
\hline & 4 & 11.17 & 9.63 & 8.96 & 8.53 & 9.55 & 23.31 & 26.21 & 20.34 & 32.33 \\
\hline & 6 & 9.66 & 9.02 & 8.07 & 8.26 & 8.98 & 15.34 & 18.01 & 14.11 & 26.31 \\
\hline \multirow[t]{3}{*}{ B6 } & 2 & 34.01 & 42.97 & 42.99 & 35.66 & 43.89 & 17.99 & 26.73 & 20.01 & 22.17 \\
\hline & 4 & 25.36 & 35.66 & 33.61 & 31.92 & 35.87 & 13.72 & 22.60 & 18.75 & 21.71 \\
\hline & 6 & 19.94 & 29.55 & 27.69 & 28.18 & 30.19 & 10.19 & 18.96 & 16.39 & 19.57 \\
\hline \multirow[t]{3}{*}{ B7 } & 2 & 100.00 & 99.99 & 89.86 & 16.23 & 99.71 & 100.00 & 100.00 & 100.00 & 100.00 \\
\hline & 4 & 100.00 & 95.87 & 41.95 & 80.41 & 82.57 & 100.00 & 100.00 & 100.00 & 100.00 \\
\hline & 6 & 100.00 & 78.12 & 34.01 & 75.80 & 60.41 & 100.00 & 76.91 & 58.36 & 100.00 \\
\hline \multirow[t]{3}{*}{ B8 } & 2 & 50.59 & 4.86 & 5.39 & 4.50 & 5.25 & 57.00 & 60.84 & 56.70 & 73.28 \\
\hline & 4 & 41.07 & 5.14 & 4.93 & 4.72 & 4.82 & 41.40 & 32.89 & 26.12 & 56.01 \\
\hline & 6 & 35.10 & 5.35 & 4.84 & 4.84 & 5.16 & 27.22 & 20.62 & 16.10 & 44.89 \\
\hline \multirow[t]{2}{*}{ B9 } & 2 & 36.94 & 10.51 & 11.33 & 9.83 & 11.33 & 19.98 & 22.28 & 18.71 & 22.89 \\
\hline & 4 & 36.05 & 10.21 & 9.64 & 9.24 & 10.19 & 13.70 & 15.65 & 12.48 & 19.25 \\
\hline
\end{tabular}

Table 8. Percentage of rejection of alternative (10) with $\theta \in\{0, .8\}$ for tests at the $5 \%$ level based on statistics (1)-(9), as estimated from 10,000 replicates of series of length $n=100$

\begin{tabular}{|c|c|c|c|c|c|c|c|}
\hline \multirow[b]{2}{*}{$\theta$} & \multirow[b]{2}{*}{ Statistic } & \multirow[b]{2}{*}{ Equation } & \multicolumn{5}{|c|}{$m$} \\
\hline & & & 2 & 3 & 4 & 5 & 6 \\
\hline \multirow[t]{9}{*}{0} & $S_{n, \hat{\sigma}}$ & (1) & 5.25 & 5.22 & 4.97 & 5.40 & 5.52 \\
\hline & $\tilde{S}_{n, .3}^{\star}$ & (3) & 4.49 & 4.39 & 4.35 & 4.51 & 4.78 \\
\hline & $\tilde{I}_{n}^{\star}$ & (5) & 4.95 & 4.71 & 4.56 & 4.83 & 4.60 \\
\hline & $\tilde{M}_{n}^{\star}$ & (7) & 4.50 & 4.45 & 4.46 & 4.78 & 4.63 \\
\hline & $\tilde{T}_{n}^{\star}$ & (9) & 4.89 & 4.82 & 4.53 & 4.80 & 4.85 \\
\hline & $\tilde{S}_{n, .3}$ & (2) & 3.86 & 4.24 & 4.24 & 4.65 & 4.26 \\
\hline & $\tilde{I}_{n}$ & (4) & 3.25 & 4.91 & 5.37 & 5.22 & 5.14 \\
\hline & $\tilde{M}_{n}$ & (6) & 4.35 & 4.69 & 5.10 & 4.94 & 5.05 \\
\hline & $\tilde{T}_{n}$ & (8) & 3.59 & 3.70 & 4.45 & 4.72 & 4.84 \\
\hline \multirow[t]{9}{*}{.8} & $S_{n, \hat{\sigma}}$ & (1) & 19.69 & 16.67 & 14.78 & 13.42 & 12.86 \\
\hline & $\tilde{S}_{n, .3}^{\star}$ & (3) & 8.20 & 8.13 & 7.65 & 7.57 & 7.57 \\
\hline & $\tilde{I}_{n}^{\star}$ & (5) & 8.84 & 8.04 & 7.84 & 7.74 & 7.31 \\
\hline & $\tilde{M}_{n}^{\star}$ & (7) & 7.76 & 7.36 & 7.62 & 7.42 & 7.26 \\
\hline & $\tilde{T}_{n}^{\star}$ & (9) & 8.93 & 8.39 & 7.95 & 7.93 & 7.94 \\
\hline & $\tilde{S}_{n, .3}$ & (2) & 23.99 & 19.22 & 15.65 & 13.38 & 11.28 \\
\hline & $\tilde{I}_{n}$ & (4) & 28.05 & 21.59 & 17.54 & 14.63 & 12.55 \\
\hline & $\tilde{M}_{n}$ & (6) & 23.05 & 16.56 & 13.66 & 11.29 & 10.21 \\
\hline & $\tilde{T}_{n}$ & (8) & 29.43 & 25.32 & 22.60 & 21.06 & 19.24 \\
\hline
\end{tabular}

embedding dimensions $m \in\{2, \ldots, 6\}$, suggesting that the logarithmic prices could be assimilated to a random walk for the period considered.

As a second illustration, consider the classic "series G" of Box, Jenkins, and Reinsel (1994), which comprises 144 monthly totals $x_{i}$ of thousands of international airline passengers. The series extends from January 1949 to December 1960. As a variance-stabilizing transformation, those authors considered the series $z_{i}=\log \left(x_{i}\right)$, which they modeled as $\nabla \nabla_{12} z_{i}=(1-\theta B)\left(1-\Theta B^{12}\right) \varepsilon_{i}$, with $\hat{\theta}=.402, \hat{\Theta}=.557$, and $\hat{\sigma}_{\varepsilon}^{2}=1.34 \times 10^{-3}$. These are maximum likelihood estimates under the Gaussian assumption. Based on the Ljung-Box statistic, Box et al. (1994) concluded that "the check does not provide any evidence of inadequacy in the model." Brockwell and Davis (1991) concurred.

The foregoing MA(1) $\times \mathrm{MA}_{12}$ (1) model satisfies Assumptions I and II, provided that second-order moments of $\varepsilon_{i}$ exist and that their density is continuous, bounded, and symmetric (as would be the case under, e.g., normality). Under these conditions, the statistics (2)-(9) provide alternative checks.

Here again, the $p$ values of statistics (2)-(9) were estimated from 10,000 replicates, and $\delta=.3$ was used for $S_{n, \hat{\sigma}}$ and $\tilde{S}_{n, \delta}^{*}$. The results (not displayed) clearly lead to rejection of the null hypothesis of independence for $m \geq 5$ consecutive innovations of the fitted model at the $5 \%$ level for all statistics except $\tilde{S}_{n, \delta}$. 
In addition, tests based on $\tilde{\mathbb{B}}_{n}^{\star}$ reject $H_{0}$ for all values of embedding dimension $m \in\{2, \ldots, 10\}$, suggesting the presence of heteroscedasticity in addition to lack of fit. When a GARCH(1, 1) model is fitted to the residuals, the GARCH coefficient is found to be significantly different from 0 . The economic implications associated with this form of heteroscedasticity are unclear, however.

\section{DISCUSSION OF THE ASSUMPTIONS}

The asymptotic results presented here depend on Assumptions I and II, and possibly also on the symmetry of $F$. Assumption I is simple and realistic. Assumption II looks more intricate, but in practice, most empirical processes based on pseudo-observations have a limit of the form specified there (see, e.g., Berkes and Horváth 2003; Ghoudi and Rémillard 2004; and references therein). The only additional restriction imposed by Assumption II is the functional independence of the term $\beta_{k}\left(x_{1}, \ldots, x_{m}\right)$ on $x_{k}$ for every $k \in\{1, \ldots, m\}$. This condition seems essential to guarantee that the asymptotic distribution of the test statistics is parameter-free.

The following result, the proof of which is given in Section B.3, gives weak regularity conditions under which Assumption II is satisfied for models of the form

$$
Y_{i}=\phi\left(\mathbf{Z}_{i-1}, \boldsymbol{\theta}\right)+\varepsilon_{i},
$$

expressed in terms of (possibly exogenous) random vectors $\mathbf{Z}_{i}$ and innovations $\varepsilon_{i}$. Here it is assumed that for $j>i$, the innovation $\varepsilon_{j}$ is independent of $\mathbf{Z}_{i}$; that $\mathbf{Z}_{1}, \mathbf{Z}_{2}, \ldots$ is a stationary and ergodic series; and that the parameter space $\mathcal{O} \subset \mathbb{R}^{d}$ is open. For example, $\operatorname{AR}(p)$ and threshold $\operatorname{AR}(p)$ models are of this form.

Lemma 1. Suppose that $F^{\prime}$ is uniformly continuous and that $\phi(\mathbf{z}, \boldsymbol{\theta})$ is continuously differentiable with respect to $\boldsymbol{\theta}$. Treat $\dot{\boldsymbol{\phi}}(\mathbf{z}, \boldsymbol{\theta})=\nabla_{\boldsymbol{\theta}} \phi(\mathbf{z}, \boldsymbol{\theta})$ as a row vector. Further assume that for any fixed $\boldsymbol{\theta} \in \mathcal{O} \subset \mathbb{R}^{d}, E\left\{\left\|\dot{\boldsymbol{\phi}}\left(\mathbf{Z}_{1}, \boldsymbol{\theta}\right)\right\|^{2}\right\}<\infty$, and that

$$
\lim _{\delta \rightarrow 0} E\left\{\sup _{\left|\boldsymbol{\theta}^{\prime}-\boldsymbol{\theta}\right|<\delta}\left\|\dot{\boldsymbol{\phi}}\left(\mathbf{Z}_{0}, \boldsymbol{\theta}^{\prime}\right)-\dot{\boldsymbol{\phi}}\left(\mathbf{Z}_{0}, \boldsymbol{\theta}\right)\right\|^{2}\right\}=0 .
$$

Suppose that $\boldsymbol{\theta}_{n}$ is an estimator of $\boldsymbol{\theta}$ such that $\boldsymbol{\Theta}_{n}=n^{1 / 2}\left(\boldsymbol{\theta}_{n}-\right.$ $\boldsymbol{\theta}) \rightsquigarrow \boldsymbol{\Theta}$ as $n \rightarrow \infty$. Let $e_{i, n}=Y_{i}-\phi\left(\mathbf{Z}_{i-1}, \boldsymbol{\theta}_{n}\right)$ denote the residuals. If $\left(\alpha_{n}, \boldsymbol{\Theta}_{n}\right) \rightsquigarrow(\alpha, \boldsymbol{\Theta})$ in $\mathcal{D}\left([-\infty, \infty]^{m}\right) \times \mathbb{R}^{d}$ as $n \rightarrow$ $\infty$, then Assumption II holds with $\beta_{k}(\mathbf{x})=-\left\{\prod_{\ell>k} F\left(x_{\ell}\right)\right\} \times$ $E\left\{\dot{\boldsymbol{\phi}}\left(\mathbf{Z}_{k-1}, \boldsymbol{\theta}\right) \prod_{\ell<k} \mathbf{1}\left(\varepsilon_{\ell} \leq x_{\ell}\right)\right\} \boldsymbol{\Theta}$ for all $\mathbf{x}=\left(x_{1}, \ldots, x_{m}\right) \in$ $[-\infty, \infty]^{m}$ and $k \in\{1, \ldots, m\}$.

As illustrated next, some—but not all—models with varying conditional variance also fall under the purview of Lemma 1.

Example 1. Consider the $\operatorname{ARCH}(p)$ model $X_{i}=(\omega+$ $\left.\sum_{j=1}^{p} a_{j} X_{i-j}^{2}\right)^{1 / 2} \epsilon_{i}$, in which the innovations $\epsilon_{i}$ are $\mathcal{N}(0,1)$ and the components of the parameter $\boldsymbol{\theta}=(\omega, \mathbf{a})$ satisfy $\omega>0$, $\mathbf{a}=\left(a_{1}, \ldots, a_{p}\right) \in[0, \infty)^{p}$ together with the second-order stationarity $a_{1}+\cdots+a_{p}<1$. Setting $Y_{i}=\log \left(X_{i}^{2}\right), \mathbf{Z}_{i-1}=$ $\left(X_{i-1}^{2}, \ldots, X_{i-p}^{2}\right)$, and $\varepsilon_{i}=\log \left(\epsilon_{i}^{2}\right)$, it follows that $Y_{i}=$ $\phi\left(\mathbf{Z}_{i-1}, \boldsymbol{\theta}\right)+\varepsilon_{i}$ for every integer $i \in \mathbb{N}$ with $\phi(\mathbf{z}, \boldsymbol{\theta})=\phi(\mathbf{z}, \omega$, a) $=\log \left(\omega+\mathbf{a}^{\top} \mathbf{z}\right)$. Note that $\varepsilon_{i}$ has density $F^{\prime}(x)=(2 \pi)^{-1 / 2} \times$ $\exp \left(x / 2-e^{x} / 2\right)$, which is uniformly continuous and squareintegrable but asymmetric. Nevertheless, the conditions of Lemma 1 are met, and thus Theorem 2 can be applied to test independence in the series $\left|\varepsilon_{i}\right|$.
Example 2. Consider the extended $\operatorname{ARCH}(p)$ model defined by $Y_{i}=X_{i}+\mu$ in which $X_{i}$ is defined as in Example 1. Using techniques from Ghoudi and Rémillard (2004), we can show that if $e_{i, n}=\left(Y_{i}-\hat{\mu}_{n}\right) /\left\{\hat{\omega}_{n}+\sum_{j=1}^{p} \hat{a}_{j n}\left(Y_{i-j}-\hat{\mu}_{n}\right)^{2}\right\}^{1 / 2}$ and $\boldsymbol{\Theta}_{n}=n^{1 / 2}\left(\hat{\mu}_{n}-\mu, \hat{\omega}_{n}-\omega, \hat{\mathbf{a}}_{n}-\mathbf{a}\right) \rightsquigarrow \boldsymbol{\Theta}=(M, \Omega, \mathbf{A})$ as $n \rightarrow \infty$, then $\mathbb{K}_{n} \rightsquigarrow \mathbb{K}$ in $\mathcal{D}\left([-\infty, \infty]^{m}\right)$, where for any $\mathbf{x}=$ $\left(x_{1}, \ldots, x_{m}\right) \in[-\infty, \infty]^{m}, \mathbb{K}(\mathbf{x})=\alpha(\mathbf{x})+\sum_{k=1}^{m} F^{\prime}\left(x_{k}\right) \beta_{k}(\mathbf{x})$ with $\beta_{k}(\mathbf{x})$ given by

$$
\begin{aligned}
& \left\{\prod_{\ell>k} F\left(x_{\ell}\right)\right\} \\
& \quad \times E\left[\left[\frac{M}{h_{k}}+\frac{x_{k}}{h_{k}^{2}}\left[\Omega+\sum_{\ell=1}^{p} A_{\ell}\left\{\left(Y_{k-\ell}-\mu-1\right)^{2}-1\right\}\right]\right]\right. \\
& \left.\quad \times \prod_{\ell<k} \mathbf{1}\left(\epsilon_{\ell} \leq x_{\ell}\right)\right],
\end{aligned}
$$

in which $h_{i}^{2}=\omega+\sum_{j=1}^{p} a_{j}\left(Y_{i-j}-\mu\right)^{2}$ for $i \in\{1, \ldots, n\}$. Assumption II is not met because $\beta_{k}$ depends on $x_{k}$ even when $\mu=0$. However, the assumption holds true when $\mu=0$ and $\varepsilon_{i}=\log \left(\left|\epsilon_{i}\right|\right)$ is considered instead of $\epsilon_{i}$.

In future work, it would be of interest to extend Lemma 1 to "recursive" models of the form $Y_{i}=\phi\left(\mathbf{Z}_{i-1}, \varepsilon_{i-1}, \ldots, \varepsilon_{i-q}\right.$, $\boldsymbol{\theta})+\varepsilon_{i}$, among which are the standard $\operatorname{ARMA}(p, q)$ models. The following result provides a partial answer when, for every $i \in \mathbb{N}$,

$$
Y_{i}-\mu-\sum_{k=1}^{p} \phi_{k}\left(Y_{i-k}-\mu\right)=\varepsilon_{i}-\sum_{j=1}^{q} \varphi_{j} \varepsilon_{i-j},
$$

where the innovations $\varepsilon_{i}$ have mean 0 and finite variance $\sigma_{\varepsilon}^{2}$, and the coefficients $\boldsymbol{\phi}=\left(\phi_{1}, \ldots, \phi_{p}\right)^{\top}$ and $\boldsymbol{\varphi}=\left(\varphi_{1}, \ldots, \varphi_{q}\right)^{\top}$ satisfy the usual conditions; that is, the roots of the polynomials $1-\sum_{k=1}^{p} \phi_{k} z^{k}$ and $1-\sum_{k=1}^{q} \varphi_{k} z^{k}$ all lie outside the unit circle. In the sequel, $\boldsymbol{\theta}_{n}=\left(\hat{\mu}_{n}, \hat{\boldsymbol{\phi}}_{n}, \hat{\boldsymbol{\varphi}}_{n}\right)$ denotes an estimation of $\boldsymbol{\theta}=$ $(\mu, \phi, \varphi)$.

Lemma 2. Suppose that for the ARMA model (12), $\left(\alpha_{n}\right.$, $\left.\boldsymbol{\Theta}_{n}\right) \rightsquigarrow(\alpha, \boldsymbol{\Theta})$ in $\mathcal{D}\left([-\infty, \infty]^{m}\right) \times \mathbb{R}^{1+p+q}$ as $n \rightarrow \infty$, and that the limit is a centered Gaussian process. If, in addition, $F^{\prime}$ is continuous and bounded, then Assumption II is satisfied.

To illustrate why the symmetry condition on $F$ is required to obtain a distribution-free limit, consider an AR(1) model with $m=2$ and centered exponential innovations, that is, $F(x)=$ $1-e^{-(1+x)}$ for $x \in[-1, \infty)$. Lemma 1 implies that Assumption II is satisfied with

$$
\begin{aligned}
\mathbb{K}\left(x_{1}, x_{2}\right)=\alpha\left(x_{1}, x_{2}\right)+F^{\prime}\left(x_{1}\right) \mathcal{P} F\left(x_{2}\right) & \\
& +F^{\prime}\left(x_{2}\right) \mathcal{P} F\left(x_{1}\right)+F^{\prime}\left(x_{2}\right) \mathcal{Q} r\left(x_{1}\right),
\end{aligned}
$$

where $r(x)=E(\varepsilon \mathbf{1}\{\varepsilon \leq x\})=-(1+x) e^{-(1+x)} \mathbf{1}(x \geq-1)$, whereas $\mathcal{P}$ and $\mathcal{Q}$ are random variables related to the asymptotic distribution of the parameters. It follows from Proposition A. 1 that the limiting copula process is of the form

$$
\mathbb{C}\left(u_{1}, u_{2}\right)=\mathbb{C}_{0}\left(u_{1}, u_{2}\right)+\mathcal{Q} F^{\prime} \circ F^{-1}\left(u_{2}\right) \times r \circ F^{-1}\left(u_{1}\right) \text {, }
$$


where

$$
\begin{aligned}
\mathbb{C}_{0}\left(u_{1}, u_{2}\right)=\alpha\left\{F^{-1}\left(u_{1}\right), F^{-1}\left(u_{2}\right)\right\} & \\
& -u_{2} \alpha\left\{F^{-1}\left(u_{1}\right), \infty\right\}-u_{1} \alpha\left\{\infty, F^{-1}\left(u_{2}\right)\right\} .
\end{aligned}
$$

Thus $\tilde{\mathbb{B}}\left(u_{1}, u_{2}\right)=\tilde{\mathbb{B}}_{0}\left(u_{1}, u_{2}\right)+\mathcal{Q} R\left(u_{1}, u_{2}\right)$, where $\tilde{\mathbb{B}}_{0}$ is the desired limiting process and, for every $u_{1}, u_{2} \in(0,1)$,

$$
\begin{aligned}
R\left(u_{1}, u_{2}\right)= & \left\{\int_{F^{-1}\left(u_{1}\right)}^{\infty} r(\xi) F^{\prime}(\xi) d \xi\right. \\
& \left.-\int_{\infty}^{F^{-1}\left(1-u_{1}\right)} r(\xi) F^{\prime}(\xi) d \xi\right\} \\
& \times\left\{\int_{F^{-1}\left(u_{2}\right)}^{\infty} F^{\prime}(\xi)^{2} d \xi-\int_{\infty}^{F^{-1}\left(1-u_{2}\right)} F^{\prime}(\xi)^{2} d \xi\right\} \\
= & \left\{1 / 4+\left(1-u_{1}\right)^{2} \log \left(1-u_{1}\right)-\left(1-u_{1}\right)^{2} / 2\right\} \\
& \times\left\{\left(1-u_{2}\right)^{2}-1 / 2\right\} .
\end{aligned}
$$

The limit $\tilde{\mathbb{B}}$ depends on $F$ and on the estimated parameters through $\mathcal{Q}$ because $R \not \equiv 0$.

\section{APPENDIX A: AUXILIARY RESULTS}

Let $\mathbf{w}_{1}, \ldots, \mathbf{w}_{n}$ be random vectors in $\mathbb{R}^{m}$, and for any $\mathbf{x}=$ $\left(x_{1}, \ldots, x_{m}\right) \in[-\infty, \infty]^{m}$ and $k \in\{1, \ldots, m\}$, set

$$
\begin{aligned}
K_{n}(\mathbf{x}) & =n^{-1} \sum_{i=1}^{n} \mathbf{1}\left(\mathbf{w}_{i} \leq \mathbf{x}\right) \quad \text { and } \\
F_{k, n}\left(x_{k}\right) & =n^{-1} \sum_{i=1}^{n} \mathbf{1}\left(w_{i k} \leq x_{k}\right) .
\end{aligned}
$$

Assume that $K_{n}$ is an estimator of an arbitrary distribution function $K$ with continuous margins $F_{1}, \ldots, F_{m}$. Then there exists a unique copula $C$ such that for all $\mathbf{x}=\left(x_{1}, \ldots, x_{m}\right) \in[-\infty, \infty]^{m}$, $K\left(x_{1}, \ldots, x_{m}\right)=C\left\{F_{1}\left(x_{1}\right), \ldots, F_{m}\left(x_{m}\right)\right\}$. Thus the empirical copula $C_{n}\left(u_{1}, \ldots, u_{m}\right)=K_{n}\left\{F_{1, n}^{-1}\left(u_{1}\right), \ldots, F_{m, n}^{-1}\left(u_{m}\right)\right\}$ estimates $C\left(u_{1}, \ldots\right.$, $\left.u_{m}\right)$ at all $\mathbf{u}=\left(u_{1}, \ldots, u_{m}\right) \in[0,1]^{m}$. For all $\mathbf{x} \in[-\infty, \infty]^{m}$, also let

$$
K^{\star}(\mathbf{x})=F_{1}\left(x_{1}\right) \cdots F_{m}\left(x_{m}\right) \text {. }
$$

Finally, assume that for each $k \in\{1, \ldots, m\}, w_{1 k}, \ldots, w_{n k}$ are mutually distinct with probability 1 . It is then a simple exercise to show that

$$
\sup _{u \in(0,1)}\left|F_{k} \circ F_{k, n}^{-1}(u)-u\right|=\sup _{u \in(0,1)}\left|F_{k, n} \circ F_{k}^{-1}(u)-u\right| .
$$

This fact is instrumental in establishing the weak convergence of the processes $\mathbb{F}_{k, n}=n^{1 / 2}\left(F_{k, n}-F_{k}\right)$ and $\mathbb{C}_{n}=n^{1 / 2}\left(C_{n}-C\right)$, which is stated next.

Proposition A.1. If $\mathbb{K}_{n}=n^{1 / 2}\left(K_{n}-K\right) \rightsquigarrow \mathbb{K}$ in $\mathcal{D}\left([-\infty, \infty]^{m}\right)$ as $n \rightarrow \infty$, then also $\mathbb{F}_{k, n} \rightsquigarrow \mathbb{F}_{k}$ in $\mathcal{D}([-\infty, \infty])$, where $\mathbb{F}_{k}\left(x_{k}\right)=$ $\mathbb{K}\left(\infty, \ldots, \infty, x_{k}, \infty, \ldots, \infty\right)$. Moreover, if $C$ has continuous derivatives of order 1 on $[0,1]^{m}$, then $\mathbb{C}_{n} \rightsquigarrow \mathbb{C}$ in $\mathcal{D}\left([0,1]^{m}\right)$ as $n \rightarrow \infty$, where $\mathbb{C}(\mathbf{u})=\mathbb{K}\left\{F_{1}^{-1}\left(u_{1}\right), \ldots, F_{m}^{-1}\left(u_{m}\right)\right\}-\sum_{k=1}^{m} \mathbb{F}_{k} \circ F_{k}^{-1}\left(u_{k}\right) \times$ $\partial C(\mathbf{u}) / \partial u_{k}$ for any $\mathbf{u}=\left(u_{1}, \ldots, u_{m}\right) \in[0,1]^{m}$.

Proof. First, the convergence of $\mathbb{F}_{k, n}$ follows from that of $\mathbb{K}_{n}$. Using (A.1) and the convergence of $\mathbb{F}_{k, n}$, we also can see that for any $k \in\{1, \ldots, m\}, \sup _{u \in(0,1)}\left|F_{k} \circ F_{k, n}^{-1}(u)-u\right| \stackrel{P}{\rightarrow} 0$ as $n \rightarrow \infty$. As a result, $\mathbb{K}_{n}\left\{F_{1, n}^{-1}\left(u_{1}\right), \ldots, F_{m, n}^{-1}\left(u_{m}\right)\right\} \rightsquigarrow \mathbb{K}\left\{F_{1}^{-1}\left(u_{1}\right), \ldots, F_{m}^{-1}\left(u_{m}\right)\right\}$ in $\mathcal{D}\left([0,1]^{m}\right)$. Next, note that for any $\mathbf{u}=\left(u_{1}, \ldots, u_{m}\right) \in[0,1]^{m}$, $\mathbb{C}_{n}(\mathbf{u})=\mathbb{K}_{n}\left\{F_{1, n}^{-1}\left(u_{1}\right), \ldots, F_{m, n}^{-1}\left(u_{m}\right)\right\}+n^{1 / 2}\left[C\left\{F_{1} \circ F_{1, n}^{-1}\left(u_{1}\right), \ldots\right.\right.$,
$\left.\left.F_{m} \circ F_{m, n}^{-1}\left(u_{m}\right)\right\}-C(\mathbf{u})\right]$. Arguments similar to those used to show (A.1) also yield the tightness of $\mathbb{Q}_{k, n}\left(u_{k}\right)=n^{1 / 2}\left\{F_{k} \circ F_{k, n}^{-1}\left(u_{k}\right)-u_{k}\right\}$ for any $k \in\{1, \ldots, m\}$. Furthermore, it is easy to check that the finite-dimensional distributions of $\mathbb{Q}_{k, n}$ converge to those of $-\mathbb{F}_{k}$ 。 $F_{k}^{-1}$. Thus we may conclude that $n^{1 / 2}\left[C\left\{F_{1} \circ F_{1, n}^{-1}\left(u_{1}\right), \ldots, F_{m}\right.\right.$ 。 $\left.\left.F_{m, n}^{-1}\left(u_{m}\right)\right\}-C(\mathbf{u})\right] \rightsquigarrow-\sum_{k=1}^{m} \mathbb{F}_{k} \circ F_{k}^{-1}\left(u_{k}\right) \partial C(\mathbf{u}) / \partial u_{k}$ as $n \rightarrow \infty$, which completes the proof.

Let $\mathcal{S}_{m}$ be the collection of all subsets of $\{1, \ldots, m\}$. For $A \in \mathcal{S}_{m}$ and $\mathbf{t}=\left(t_{1}, \ldots, t_{m}\right) \in[-\infty, \infty]^{m}$, let $\mathbf{t}_{A}$ denote an $m$-dimensional vector, the $k$ th component of which equals $-t_{k}$ if $k \in A$ and $t_{k}$ otherwise. For arbitrary $\mathbf{t} \in[0, \infty]^{m}$, let

$$
B_{n}(\mathbf{t})=n^{-2} \sum_{i=1}^{n} \sum_{j=1}^{n} \prod_{k=1}^{m} \mathbf{1}\left(\left|w_{j k}-w_{i k}\right| \leq t_{k}\right) .
$$

Write $\psi(h)(\mathbf{t})=\sum_{A \in S_{m}}(-1)^{|A|} \int h\left(x+\mathbf{t}_{A}\right) d K(\mathbf{x})$ for any $h \in$ $\mathcal{D}\left([-\infty, \infty]^{m}\right)$, and define $\psi^{\star}(h)$ similarly, with $K^{\star}$ instead of $K$. Both $\psi$ and $\psi^{\star}$ are continuous linear mappings from $\mathcal{D}\left([-\infty, \infty]^{m}\right)$ to $\mathcal{D}\left([0, \infty]^{m}\right)$. Furthermore, set $\mathbb{B}_{n}=n^{1 / 2}\left(B_{n}-B\right)$, where, for arbitrary $\mathbf{t} \in[0, \infty]^{m}$,

$$
B(\mathbf{t})=\psi(K)(\mathbf{t})=\sum_{A \in \mathcal{S}_{m}}(-1)^{|A|} \int_{\mathbb{R}^{m}} K\left(\mathbf{x}+\mathbf{t}_{A}\right) d K(\mathbf{x}) .
$$

Next, let $\mathbb{B}_{n}^{\star}=2 \psi^{\star}\left(\mathbb{K}_{n}\right)$. Using the multinomial formula $\prod_{k=1}^{m}\left(x_{k}+y_{k}\right)=\sum_{A \in \mathcal{S}_{m}}\left(\prod_{k \in A} x_{k}\right) \times\left(\prod_{\ell \notin A} y_{\ell}\right)$, we can check that for all $\mathbf{t}=\left(t_{1}, \ldots, t_{m}\right) \in[0, \infty]^{m}$,

$$
\begin{aligned}
\mathbb{B}_{n}^{\star}(\mathbf{t})=2 n^{-1 / 2} & \\
\times & \sum_{i=1}^{n}\left[\prod_{k=1}^{m}\left\{F_{k}\left(w_{i k}+t_{k}\right)-F_{k}\left(w_{i k}-t_{k}\right)\right\}-\prod_{k=1}^{m} G_{k}\left(t_{k}\right)\right],
\end{aligned}
$$

where $G_{k}\left(t_{k}\right)=\int\left\{F_{k}\left(\xi+t_{k}\right)-F_{k}\left(\xi-t_{k}\right)\right\} d F_{k}(\xi)$ for all $k \in$ $\{1, \ldots, m\}$.

We give the asymptotic behaviors of $\mathbb{B}_{n}$ and $\mathbb{B}_{n}^{\star}$ next.

Proposition A.2. If $\mathbb{K}_{n} \rightsquigarrow \mathbb{K}$ in $\mathcal{D}\left([-\infty, \infty]^{m}\right)$ as $n \rightarrow \infty$, then $\sup _{\mathbf{t} \in[0, \infty]^{m}}\left|\mathbb{B}_{n}(\mathbf{t})-2 \psi\left(\mathbb{K}_{n}\right)(\mathbf{t})\right| \stackrel{P}{\rightarrow} 0, \mathbb{B}_{n} \rightsquigarrow \mathbb{B}=2 \psi(\mathbb{K})$, and $\mathbb{B}_{n}^{\star} \rightsquigarrow$ $\mathbb{B}^{\star}=2 \psi^{\star}(\mathbb{K})$ in $\mathcal{D}\left([0, \infty]^{m}\right)$ as $n \rightarrow \infty$. Moreover, $\gamma_{n} \stackrel{P}{\rightarrow} \gamma$ as $n \rightarrow$ $\infty$

Proof. First, it follows from the weak convergence of $\mathbb{K}_{n}$ to $\mathbb{K}$ that, as $n \rightarrow \infty$,

$$
\sup _{\mathbf{t} \in[0, \infty]^{m}}\left|\frac{1}{n} \sum_{i=1}^{n} \mathbb{K}_{n}\left(\mathbf{w}_{i}+\mathbf{t}\right)-\int_{\mathbb{R}^{m}} \mathbb{K}_{n}(\mathbf{x}+\mathbf{t}) d K(\mathbf{x})\right| \stackrel{P}{\rightarrow} 0 .
$$

Next, an application of the multinomial formula yields

$$
\begin{aligned}
B_{n}(\mathbf{t})= & n^{-2} \sum_{i=1}^{n} \sum_{j=1}^{n} \prod_{k=1}^{m} \mathbf{1}\left(\left|w_{j k}-w_{i k}\right| \leq t_{k}\right) \\
= & n^{-2} \sum_{A \in \mathcal{S}_{m}}(-1)^{|A|} \sum_{i=1}^{n} \sum_{j=1}^{n}\left\{\prod_{k \in A} \mathbf{1}\left(w_{i k}<w_{j k}-t_{k}\right)\right\} \\
& \times \prod_{k \in \mathcal{S}_{m} \backslash A} \mathbf{1}\left(w_{i k} \leq w_{j k}+t_{k}\right),
\end{aligned}
$$

and thus for all $\mathbf{t}=\left(t_{1}, \ldots, t_{m}\right) \in[0, \infty]^{m}$, we have

$$
B_{n}(\mathbf{t})=n^{-2} \sum_{A \in \mathcal{S}_{m}}(-1)^{|A|} \sum_{i=1}^{n} \sum_{j=1}^{n} \mathbf{1}\left(\mathbf{w}_{i} \leq \mathbf{w}_{j}+\mathbf{t}_{A}\right)+o_{P}\left(n^{-1 / 2}\right)
$$




$$
\begin{aligned}
= & n^{-1} \sum_{A \in \mathcal{S}_{m}}(-1)^{|A|} \sum_{j=1}^{n} K_{n}\left(\mathbf{w}_{j}+\mathbf{t}_{A}\right)+o_{P}\left(n^{-1 / 2}\right) \\
= & n^{-3 / 2} \sum_{A \in \mathcal{S}_{m}}(-1)^{|A|} \sum_{j=1}^{n} \mathbb{K}_{n}\left(\mathbf{w}_{j}+\mathbf{t}_{A}\right) \\
& +n^{-1} \sum_{A \in \mathcal{S}_{m}}(-1)^{|A|} \sum_{j=1}^{n} K\left(\mathbf{w}_{j}+\mathbf{t}_{A}\right)+o_{P}\left(n^{-1 / 2}\right) .
\end{aligned}
$$

Furthermore, the identity

$$
\begin{aligned}
\sum_{A \in S_{m}}(-1)^{|A|} \mathbf{1}\left(\mathbf{x} \leq \mathbf{w}_{j}+\mathbf{t}_{A}\right) & =\mathbf{1}\left\{\left|\mathbf{x}-\mathbf{w}_{j}\right| \leq \mathbf{t}\right\} \\
& =\sum_{A \in S_{m}}(-1)^{|A|} \mathbf{1}\left(\mathbf{w}_{j} \leq \mathbf{x}+\mathbf{t}_{A}\right)
\end{aligned}
$$

holds almost surely for all $\mathbf{x} \in[-\infty, \infty]^{m}$ and $\mathbf{t}=\left(t_{1}, \ldots, t_{m}\right) \in$ $[0, \infty]^{m}$. Therefore,

$$
\begin{aligned}
n^{-1} & \sum_{A \in \mathcal{S}_{m}}(-1)^{|A|} \sum_{j=1}^{n} K\left(\mathbf{w}_{j}+\mathbf{t}_{A}\right) \\
= & n^{-1} \sum_{j=1}^{n} \sum_{A \in \mathcal{S}_{m}}(-1)^{|A|} \int_{\mathbb{R}^{m}} \mathbf{1}\left(\mathbf{x} \leq \mathbf{w}_{j}+\mathbf{t}_{A}\right) d K(\mathbf{x}) \\
= & \sum_{A \in \mathcal{S}_{m}}(-1)^{|A|} \int_{\mathbb{R}^{m}} K_{n}\left(\mathbf{x}+\mathbf{t}_{A}\right) d K(\mathbf{x})
\end{aligned}
$$

can be expressed differently as

$$
\begin{aligned}
n^{-1} & \sum_{A \in \mathcal{S}_{m}}(-1)^{|A|} \sum_{j=1}^{n} K\left(\mathbf{w}_{j}+\mathbf{t}_{A}\right) \\
= & n^{-1 / 2} \sum_{A \in S_{m}}(-1)^{|A|} \int_{\mathbb{R}^{m}} \mathbb{K}_{n}\left(\mathbf{x}+\mathbf{t}_{A}\right) d K(\mathbf{x}) \\
& +\sum_{A \in \mathcal{S}_{m}}(-1)^{|A|} \int_{\mathbb{R}^{m}} K\left(\mathbf{x}+\mathbf{t}_{A}\right) d K(\mathbf{x}) \\
= & n^{-1 / 2} \sum_{A \in \mathcal{S}_{m}}(-1)^{|A|} \int_{\mathbb{R}^{m}} \mathbb{K}_{n}\left(\mathbf{x}+\mathbf{t}_{A}\right) d K(\mathbf{x})+B(\mathbf{t}) .
\end{aligned}
$$

Thus the following chain of identities holds uniformly in $\mathbf{t} \in[0, \infty]^{m}$ :

$$
\begin{aligned}
\mathbb{B}_{n}(\mathbf{t})= & n^{-1} \sum_{A \in \mathcal{S}_{m}}(-1)^{|A|} \sum_{j=1}^{n} \mathbb{K}_{n}\left(\mathbf{w}_{j}+\mathbf{t}_{A}\right) \\
& +\sum_{A \in \mathcal{S}_{m}}(-1)^{|A|} \int_{\mathbb{R}^{m}} \mathbb{K}_{n}\left(\mathbf{x}+\mathbf{t}_{A}\right) d K(\mathbf{x})+o_{P}(1) \\
= & 2 \sum_{A \in \mathcal{S}_{m}}(-1)^{|A|} \int_{\mathbb{R}^{m}} \mathbb{K}_{n}\left(\mathbf{x}+\mathbf{t}_{A}\right) d K(\mathbf{x})+o_{P}(1) \\
= & 2 \psi\left(\mathbb{K}_{n}\right)(\mathbf{t})+o_{P}(1)
\end{aligned}
$$

The continuous mapping theorem then implies that $\psi\left(\mathbb{K}_{n}\right) \rightsquigarrow \psi(\mathbb{K})$ and $\psi^{\star}\left(\mathbb{K}_{n}\right) \rightsquigarrow \psi^{\star}(\mathbb{K})$ in $\mathcal{D}\left([0, \infty]^{m}\right)$ as $n \rightarrow \infty$.

Finally, to establish the consistency of $\gamma_{n}$ as an estimate of $\gamma$, introduce independent variables $\tilde{\epsilon}_{1}$ and $\tilde{\epsilon}_{2}$ distributed as $F$ and write $F_{n}(s)=K_{n}(s, \infty, \ldots, \infty)$ for all $s \in \mathbb{R}$. It follows from the uniform convergence of $F_{n}$ to $F$ that for all $u, v \in[0, \infty)$,

$$
\gamma_{n}(u, v)=n^{-1} \sum_{k=1}^{n}\left\{F_{n}\left(e_{k}+u\right)-F_{n}\left(e_{k}-u\right)\right\}
$$

$$
\begin{aligned}
& \times\left\{F_{n}\left(e_{k}+v\right)-F_{n}\left(e_{k}-v\right)\right\}+o_{P}(1) \\
= & n^{-1} \sum_{k=1}^{n}\left\{F\left(e_{k}+u\right)-F\left(e_{k}-u\right)\right\} \\
& \times\left\{F\left(e_{k}+v\right)-F\left(e_{k}-v\right)\right\}+o_{P}(1) \\
= & n^{-1} \sum_{k=1}^{n} E\left[\mathbf{1}\left\{\left|e_{k}-\tilde{\epsilon}_{1}\right| \leq u\right\} \mathbf{1}\left\{\left|e_{k}-\tilde{\epsilon}_{2}\right| \leq v\right\}\right]+o_{P}(1) \\
= & E\left[F_{n}\left\{\left(\tilde{\epsilon}_{1}+u\right) \wedge\left(\tilde{\epsilon}_{2}+v\right)\right\}-F_{n}\left\{\left(\tilde{\epsilon}_{1}-u\right) \vee\left(\tilde{\epsilon}_{2}-v\right)\right\}\right] \\
& +o o_{P}(1) \\
= & \gamma(u, v)+o_{P}(1),
\end{aligned}
$$

because $\gamma(u, v)=E\left[F\left\{\left(\tilde{\epsilon}_{1}+u\right) \wedge\left(\tilde{\epsilon}_{2}+v\right)\right\}-F\left\{\left(\tilde{\epsilon}_{1}-u\right) \vee\left(\tilde{\epsilon}_{2}-v\right)\right\}\right]$ by definition.

We can now state the main result of this appendix.

Proposition A.3. Suppose that the margins $F_{1}, \ldots, F_{m}$ of $K$ admit continuous and square-integrable derivatives $F_{1}^{\prime}, \ldots, F_{m}^{\prime}$. Further, assume that $K=K^{\star}$ and that there exist processes $\alpha, \beta_{1}, \ldots, \beta_{m} \in$ $\mathcal{D}\left([-\infty, \infty]^{m}\right)$ such that for all $\mathbf{x}=\left(x_{1}, \ldots, x_{m}\right) \in[-\infty, \infty]^{m}$, $\beta_{k}(\mathbf{x})$ does not depend on $x_{k}$ for every $k \in\{1, \ldots, m\}$. Finally, suppose that $\mathbb{K}_{n} \rightsquigarrow \mathbb{K}$ in $\mathcal{D}\left([-\infty, \infty]^{m}\right)$ as $n \rightarrow \infty$, where for all $\mathbf{x} \in[-\infty$, $\infty]^{m}, \mathbb{K}(\mathbf{x})=\alpha(\mathbf{x})-\sum_{k=1}^{m} F_{k}^{\prime}\left(x_{k}\right) \beta_{k}(\mathbf{x})$. Then $\sup _{\mathbf{t} \in \mathbb{R}^{m}} \mid \mathbb{B}_{n}(\mathbf{t})-$ $\mathbb{B}_{n}^{\star}(\mathbf{t}) \mid \stackrel{P}{\rightarrow} 0$ and $\mathbb{B}_{n} \rightsquigarrow \mathbb{B}=2 \psi(\alpha)$ in $\mathcal{D}\left([0, \infty]^{m}\right)$ as $n \rightarrow \infty$.

Proof. We have $\psi^{\star}=\psi$ by hypothesis, and thus $\sup _{\mathbf{t} \in \mathbb{R}^{m}} \mid \mathbb{B}_{n}(\mathbf{t})-$ $\mathbb{B}_{n}^{\star}(\mathbf{t}) \mid \stackrel{P}{\rightarrow} 0$ and $\mathbb{B}_{n} \rightsquigarrow \mathbb{B}=2 \psi(\mathbb{K})=2 \psi(\alpha)-2 \sum_{k=1}^{m} \psi\left(F_{k}^{\prime} \beta_{k}\right)$ as $n \rightarrow \infty$ by Proposition A.2. Thus it only remains to show that for any $k \in\{1, \ldots, m\}, \psi\left(F_{k}^{\prime} \beta_{k}\right) \equiv 0$. The argument being the same for each $k$, take $k=m$ for the sake of simplicity. Then, for any $\mathbf{t}=\left(t_{1}, \ldots, t_{m}\right) \in$ $[0, \infty]^{m}$,

$$
\begin{aligned}
& \psi\left(F_{m}^{\prime} \beta_{m}\right)(\mathbf{t}) \\
& =\sum_{A \in \mathcal{S}_{m}}(-1)^{|A|} \int_{\mathbb{R}^{m}} F_{m}^{\prime}\left\{x_{m}+\left(\mathbf{t}_{A}\right)_{m}\right\} \beta_{m}\left(\mathbf{x}+\mathbf{t}_{A}\right) d K(\mathbf{x}) \\
& =\sum_{A \in \mathcal{S}_{m-1}}(-1)^{|A|}\left\{\int _ { \mathbb { R } ^ { m } } \left\{F_{m}^{\prime}\left(x_{m}+t_{m}\right)\right.\right. \\
& \left.\left.\quad-F_{m}^{\prime}\left(x_{m}-t_{m}\right)\right\} d F_{m}\left(x_{m}\right)\right\} \\
& \quad \times \int_{\mathbb{R}^{m}} \beta_{m}\left(\mathbf{x}+\mathbf{t}_{A}\right) d F_{1}\left(x_{1}\right) \cdots d F_{m-1}\left(x_{m-1}\right),
\end{aligned}
$$

because $\beta_{m}(\mathbf{x})$ does not depend on $x_{m}$. That the entire expression vanishes then follows from the fact that, in view of the square-integrability of $F_{m}^{\prime}$,

$$
\int_{-\infty}^{\infty}\left\{F_{m}^{\prime}\left(\xi+t_{m}\right)-F_{m}^{\prime}\left(\xi-t_{m}\right)\right\} d F_{m}(\xi)=0
$$

Now, for every $k \in\{1, \ldots, m\}$ and $\mathbf{t}=\left(t_{1}, \ldots, t_{m}\right) \in[0, \infty]^{m}$, set $G_{k, n}\left(t_{k}\right)=B_{n}\left(\infty, \ldots, \infty, t_{k}, \infty, \ldots, \infty\right)$ and define $\mathbb{D}_{n}(\mathbf{t})=n^{1 / 2} \times$ $\left\{B_{n}(\mathbf{t})-\prod_{k=1}^{m} G_{k, n}\left(t_{k}\right)\right\}$. As shown later, the weak convergence of the process $\mathbb{D}_{n}$ is then a consequence of the previous result. Before stating this fact precisely, define $\psi_{k}$ for any $k \in\{1, \ldots, m\}$ by $\psi_{k}(h)\left(t_{k}\right)=\int\left\{h\left(\xi+t_{k}\right)-h\left(\xi-t_{k}\right)\right\} d F_{k}(\xi)$ for all $t_{k} \in[0, \infty]$ and $h \in \mathcal{D}([-\infty, \infty])$.

Corollary A.1. Suppose that the conditions of Proposition A.3 hold true. For every $k \in\{1, \ldots, m\}$ and $\mathbf{x}=\left(x_{1}, \ldots, x_{m}\right) \in[-\infty, \infty]^{m}$, set $\alpha_{k}\left(x_{k}\right)=\alpha\left(\infty, \ldots, \infty, x_{k}, \infty, \ldots, \infty\right)$. Then $\mathbb{D}_{n} \rightsquigarrow \mathbb{D}$ in $\mathcal{D}\left([0, \infty]^{m}\right)$ as $n \rightarrow \infty$, and for all $k \in\{1, \ldots, m\}, \mathbb{G}_{k, n}=n^{1 / 2}\left(G_{k, n}-G_{k}\right) \rightsquigarrow$ 
$\mathbb{G}_{k}=2 \psi_{k}\left(\alpha_{k}\right)$ in $\mathcal{D}([0, \infty])$ as $n \rightarrow \infty$, where, for every $\mathbf{t}=\left(t_{1}\right.$, $\left.\ldots, t_{m}\right) \in[0, \infty]^{m}, \mathbb{D}=2 \psi(\alpha)-2 \sum_{k=1}^{m} \psi_{k}\left(\alpha_{k}\right)\left(t_{k}\right) \prod_{j \neq k} G_{j}\left(t_{j}\right)$.

Proof. First, note that for every $k \in\{1, \ldots, m\}$ and $\mathbf{x}=\left(x_{1}, \ldots\right.$, $\left.x_{m}\right) \in[-\infty, \infty]^{m}, \mathbb{F}_{k, n} \rightsquigarrow \mathbb{F}_{k}\left(x_{k}\right)=\alpha_{k}\left(x_{k}\right)-F_{k}^{\prime}\left(x_{k}\right) \beta_{k}(\infty, \ldots, \infty)$ as $n \rightarrow \infty$. Proposition A.2 implies that $\mathbb{G}_{k, n}\left(t_{k}\right)=2 \psi_{k}\left(\mathbb{F}_{k, n}\right)\left(t_{k}\right)+$ $o_{P}(1)$, so $\mathbb{G}_{k, n} \rightsquigarrow 2 \psi_{k}\left(\mathbb{F}_{k}\right)=2 \psi_{k}\left(\alpha_{k}\right)$ in $\mathcal{D}([0, \infty])$, because of (A.2). To conclude, note that $\mathbb{D}_{n}(\mathbf{t})$ equals

$$
\begin{aligned}
\mathbb{B}_{n}(\mathbf{t})+n^{1 / 2}\left\{\prod_{k=1}^{m} G_{k, n}\left(t_{k}\right)-B(\mathbf{t})\right\} & \\
= & \mathbb{B}_{n}(\mathbf{t})+\sum_{k=1}^{m} \mathbb{G}_{k, n}\left(t_{k}\right)\left\{\prod_{j \neq k} G_{j}\left(t_{j}\right)\right\}
\end{aligned}
$$

up to an additive term that is $o_{P}(1)$ uniformly in $\mathbf{t} \in[0, \infty]^{m}$.

Next, we consider the weak convergence of rank-based analogs of $\mathbb{D}_{n}$ and $\mathbb{B}_{n}$. Toward this end, let $\tilde{\mathbf{w}}_{i}=\left(\tilde{w}_{i 1}, \ldots, \tilde{w}_{i m}\right)=$ $\left(F_{1, n}\left(w_{i 1}\right), \ldots, F_{m, n}\left(w_{i m}\right)\right)$ for all $i \in\{1, \ldots, n\}$. Note that $C_{n}(\mathbf{t})=$ $\sum_{i=1}^{n} \mathbf{1}\left(\tilde{\mathbf{w}}_{i} \leq \mathbf{t}\right) / n$ for all $\mathbf{t} \in[-\infty, \infty]^{m}$. For all $\mathbf{u} \in[0,1]^{m}$, let

$$
\tilde{B}_{n}(\mathbf{u})=n^{-2} \sum_{i=1}^{n} \sum_{j=1}^{n} \mathbf{1}\left(\left\|\tilde{\mathbf{w}}_{i}-\tilde{\mathbf{w}}_{j}\right\| \leq \mathbf{u}\right) .
$$

Further, define the mappings $\tilde{\psi}: h \in \mathcal{D}\left([-\infty, \infty]^{m}\right) \mapsto \tilde{\psi}(h) \in$ $\mathcal{D}\left([0,1]^{m}\right)$ and $\tilde{\psi}_{1}: h_{1} \in \mathcal{D}([-\infty, \infty]) \mapsto \tilde{\psi}_{1}\left(h_{1}\right) \in \mathcal{D}([0,1])$ by

$$
\tilde{\psi}(h)(\mathbf{u})=\sum_{A \in \mathcal{S}_{m}}(-1)^{|A|} \int_{[0,1]^{m}} h\left(\mathbf{x}+\mathbf{u}_{A}\right) d \mathbf{x}
$$

and

$$
\tilde{\psi}_{1}\left(h_{1}\right)\left(u_{1}\right)=\int_{0}^{1}\left\{h_{1}\left(s+u_{1}\right)-h_{1}\left(s-u_{1}\right)\right\} d s .
$$

Next, set $\tilde{\mathbb{B}}_{n}^{\star}=2 \tilde{\psi}\left(\mathbb{C}_{n}\right)$ and $\tilde{B}(\mathbf{u})=\prod_{k=1}^{m} \tilde{G}\left(u_{k}\right)$. Then, for every $\mathbf{u} \in[0,1]^{m}$,

$$
\begin{aligned}
\tilde{\mathbb{B}}_{n}^{\star}(\mathbf{u}) & =2 \tilde{\psi}\left(\mathbb{C}_{n}\right)(\mathbf{u})=2 \sum_{A \in \mathcal{S}_{m}}(-1)^{|A|} \int_{[0,1]^{m}} \mathbb{C}_{n}\left(\mathbf{x}+\mathbf{u}_{A}\right) d \mathbf{x} \\
& =2 n^{-1 / 2} \sum_{i=1}^{n}\left[\prod_{k=1}^{m}\left\{\tilde{F}\left(\tilde{w}_{i k}+u_{k}\right)-\tilde{F}\left(\tilde{w}_{i k}-u_{k}\right)\right\}-\tilde{B}(\mathbf{u})\right],
\end{aligned}
$$

with $\tilde{F}(x)=P\left(U_{1} \leq x\right)$ for every $x \in \mathbb{R}$ and $\tilde{G}(s)=P\left(\left|U_{2}-U_{1}\right| \leq s\right)$ $\left.=\psi_{1}(\tilde{F})(s)=\int \tilde{F}(u+s)-\tilde{F}(u-s)\right\} d u=2 s-s^{2}$ for all $s \in[0,1]$, where $U_{1}$ and $U_{2}$ are $\operatorname{Unif}(0,1)$ and independent. Finally, set $\tilde{\mathbb{B}}_{n}=$ $n^{1 / 2}\left(\tilde{B}_{n}-\tilde{B}\right)$.

The stage is now set for the final result of this appendix.

Corollary A.2. Assume that the conditions of Proposition A.3 hold. For every $k \in\{1, \ldots, m\}$, let $\alpha_{k}$ be defined as in Corollary A.1. For all $\mathbf{u}=\left(u_{1}, \ldots, u_{m}\right) \in[0,1]^{m}$, set $\tilde{\alpha}_{k}\left(u_{k}\right)=\alpha_{k} \circ F_{k}^{-1}\left(u_{k}\right)$ and define $\tilde{\alpha}(\mathbf{u})=\alpha\left\{F_{1}^{-1}\left(u_{1}\right), \ldots, F_{m}^{-1}\left(u_{m}\right)\right\}$. Then $\sup _{\mathbf{u} \in[0,1]^{m}} \mid \tilde{\mathbb{B}}_{n}(\mathbf{u})-$ $\tilde{\mathbb{B}}_{n}^{\star}(\mathbf{u}) \mid \stackrel{P}{\rightarrow} 0$ and $\tilde{\mathbb{B}}_{n} \rightsquigarrow \tilde{\mathbb{B}}=2 \tilde{\psi}(\mathbb{C})$ in $\mathcal{D}\left([0,1]^{m}\right)$ as $n \rightarrow \infty$. If $\mathbb{K} \neq$ $\alpha$, then further assume that $F_{1}, \ldots, F_{m}$ are symmetric. Then, for all $\mathbf{u} \in[0,1]^{m}, \tilde{\mathbb{B}}(\mathbf{u})=2 \tilde{\psi}(\tilde{\alpha})(\mathbf{u})-2 \sum_{k=1}^{m} \tilde{\psi}_{1}\left(\tilde{\alpha}_{k}\right)\left(u_{k}\right) \prod_{j \neq k} \tilde{G}_{j}\left(u_{j}\right)$.

Proof. Fix $\mathbf{u}=\left(u_{1}, \ldots, u_{m}\right) \in[0,1]^{m}$. First, note that from Lemma A.1, $\mathbb{C}_{n} \rightsquigarrow \mathbb{C}$ as $n \rightarrow \infty$. Using the representation in Lemma 1, we find that

$$
\begin{aligned}
\mathbb{C}(\mathbf{u})= & \mathbb{K}\left\{F_{1}^{-1}\left(u_{1}\right), \ldots, F_{m}^{-1}\left(u_{m}\right)\right\} \\
& -\sum_{k=1}^{m} \mathbb{F}_{k} \circ F_{k}^{-1}\left(u_{k}\right)\left(\frac{\partial}{\partial u_{k}} \prod_{j=1}^{m} u_{j}\right)
\end{aligned}
$$

$$
\begin{aligned}
= & \tilde{\alpha}(\mathbf{u})-\sum_{k=1}^{m} \tilde{\alpha}_{k}\left(u_{k}\right) \prod_{j \neq k} u_{j} \\
& -\sum_{k=1}^{m} F_{k}^{\prime} \circ F_{k}^{-1}\left(u_{k}\right)\left\{\tilde{\beta}_{k}(\mathbf{u})-\tilde{\beta}_{k}(1, \ldots, 1) \prod_{j \neq k} u_{j}\right\},
\end{aligned}
$$

with $\tilde{\beta}_{k}(\mathbf{u})=\beta_{k}\left\{F_{1}^{-1}\left(u_{1}\right), \ldots, F_{m}^{-1}\left(u_{m}\right)\right\}$. From Proposition A.2, $\sup _{\mathbf{u} \in[0,1]^{m}}\left|\tilde{\mathbb{B}}_{n}(\mathbf{u})-\tilde{\mathbb{B}}_{n}^{\star}(\mathbf{u})\right| \stackrel{P}{\rightarrow} 0$ and $\mathbb{B}_{n} \rightsquigarrow \mathbb{B}=2 \tilde{\psi}(\mathbb{C})$ in $\mathcal{D}\left([0,1]^{m}\right)$ as $n \rightarrow \infty$.

If $\mathbb{K}=\alpha$, then the proof is complete. Otherwise, because $\tilde{\beta}_{k}(\mathbf{u})$ does not depend on $u_{k}$, it remains to show that $\tilde{\psi}_{1}\left(F_{k}^{\prime} \circ F_{k}^{-1}\right) \equiv 0$ on $[0,1]$ for all $k \in\{1, \ldots, m\}$. Toward this end, note that for all $x \in(0,1)$, we have

$$
\begin{aligned}
& \tilde{\psi}_{1}\left(F_{k}^{\prime} \circ F_{k}^{-1}\right)(x) \\
& =\int_{0}^{1}\left\{F_{k}^{\prime} \circ F_{k}^{-1}(\xi+x)-F_{k}^{\prime} \circ F_{k}^{-1}(\xi-x)\right\} d \xi, \\
& =\int_{x}^{1} F_{k}^{\prime} \circ F_{k}^{-1}(\xi) d \xi-\int_{0}^{1-x} F_{k}^{\prime} \circ F_{k}^{-1}(\xi) d \xi \\
& =\int_{F_{k}^{-1}(x)}^{\infty}\left\{F_{k}^{\prime}(\xi)\right\}^{2} d \xi-\int_{-\infty}^{F_{k}^{-1}(1-x)}\left\{F_{k}^{\prime}(\xi)\right\}^{2} d \xi
\end{aligned}
$$

Set $Q_{k}(u)=F_{k}^{-1}(u)$ for $u \in(0,1)$. Then $Q_{k}^{\prime}(u)=1 / F_{k}^{\prime} \circ F_{k}^{-1}(u)$, so that $\tilde{\psi}_{1}\left(F_{k}^{\prime} \circ F_{k}^{-1}\right) \equiv 0 \Leftrightarrow Q_{k}^{\prime}(u)=Q_{k}^{\prime}(1-u)$ for all $u \in(0,1)$, demonstrating that $F_{k}$ is symmetric.

\section{APPENDIX B: PROOFS OF THE MAIN RESULTS}

Given that Theorem 1 is a special case of Theorem 3, only the latter is proved here. Further note that although it is convenient to define the last $m-1$ values of $\tilde{e}_{i}$ in a circular way, as was done in Section 2, this does not affect the asymptotic distribution of any statistic based on $\tilde{e}_{1}, \ldots, \tilde{e}_{n+m-1}$. Therefore, the arguments herein are presented as if a sample of size $n+m-1$ (rather than $n$ ) had been collected.

\section{B.1 Proof of Theorem 2}

Proposition A.3 implies that as $n \rightarrow \infty,\left(\mathbb{B}_{n}, \mathbb{B}_{n}^{\star}\right) \rightsquigarrow(\mathbb{B}, \mathbb{B})$ with $\mathbb{B}=2 \psi(\alpha)$. Because $\mathbb{D}_{n}$ is a continuous functional of $\mathbb{B}_{n}$ (see the proof of Corollary A.1), we also get $\left(\mathbb{B}_{n}, \mathbb{B}_{n}^{\star}, \mathbb{D}_{n}\right) \rightsquigarrow(\mathbb{B}, \mathbb{B}, \mathbb{D})$ as $n \rightarrow \infty$.

To compute the covariance $\Gamma_{\mathbb{B}}$, use the fact that because $\alpha_{n} \rightsquigarrow \alpha$ as $n \rightarrow \infty$,

$$
\begin{aligned}
\overline{\mathbb{B}}_{n}^{\star}(\mathbf{t})= & 2 \psi\left(\alpha_{n}\right)(\mathbf{t}) \\
= & 2 n^{-1 / 2} \sum_{i=1}^{n}\left[\prod_{k=1}^{m}\left\{F\left(\varepsilon_{i+k-1}+t_{k}\right)-F\left(\varepsilon_{i+k-1}-t_{k}\right)\right\}\right. \\
& \left.-\prod_{k=1}^{m} G\left(t_{k}\right)\right]
\end{aligned}
$$

has the same limiting covariance as $\mathbb{B}$. As for $\Gamma_{\mathbb{D}}$, its formula stems from the representation of $\mathbb{D}$ in Corollary A.1, together with the fact that $\operatorname{cov}\left\{\psi_{1}\left(\alpha_{1}\right)(s), \psi(\alpha)(\mathbf{t})\right\}=\operatorname{cov}\left\{\psi_{1}\left(\alpha_{1}\right)(s), \psi_{1}\left(\alpha_{1}\right)\left(t_{1}\right)\right\}=$ $\gamma\left(s, t_{1}\right)-G(s) G\left(t_{1}\right)$. Finally, the validity of Algorithm 1 is a consequence of the fact that $\overline{\mathbb{B}}_{n}=n^{1 / 2}\left(\bar{B}_{n}-B\right) \rightsquigarrow \mathbb{B}$, where $\bar{B}_{n}(\mathbf{t})=n^{-2} \sum_{i=1}^{n} \sum_{j=1}^{n} \prod_{k=1}^{m} \mathbf{1}\left(\left|\varepsilon_{j+k-1}-\varepsilon_{i+k-1}\right| \leq t_{k}\right)$ for all $\mathbf{t}=\left(t_{1}, \ldots, t_{m}\right) \in[0, \infty]^{m}$. 


\section{B.2 Proof of Theorem 3}

As mentioned just before Theorem $3, \sup _{\mathbf{t} \in[0,1]^{m}}\left|\tilde{\mathbb{D}}_{n}(\mathbf{t})-\tilde{\mathbb{B}}(\mathbf{t})\right|=$ $O\left(n^{-1}\right)$. Thus it follows from Corollary A.2 that $\left(\tilde{\mathbb{B}}_{n}, \tilde{\mathbb{B}}_{n}^{\star}, \tilde{\mathbb{D}}_{n}\right) \rightsquigarrow(\tilde{\mathbb{B}}$, $\tilde{\mathbb{B}}, \tilde{\mathbb{B}})$ as $n \rightarrow \infty$. Given that $\tilde{\mathbb{B}}$ has the same form as $\mathbb{D}$ when $F$ and $G$ are replaced by $\tilde{F}$ and $\tilde{G}$, the covariance formula given in Theorem 2 remains true. The validity of Algorithm 2 stems from the fact that $\tilde{\alpha}_{n} \rightsquigarrow \tilde{\alpha}$ as $n \rightarrow \infty$, together with the fact that $\tilde{\mathbb{B}}$ depends only on $\tilde{\alpha}$.

\section{B.3 Proof of Lemma 1}

First, note that the weak convergence of $\mathbb{K}_{n}$ on $\mathcal{D}\left([-\infty, \infty]^{m}\right)$ is equivalent to the statement $\mathbb{E}_{n}=\mathbb{K}_{n}\left(F^{-1}, \ldots, F^{-1}\right) \rightsquigarrow \mathbb{E}=$ $\mathbb{K}\left(F^{-1}, \ldots, F^{-1}\right)$ on $\mathcal{D}\left([0,1]^{m}\right)$. For each $i \in\{1, \ldots, n\}$, introduce $U_{i}=F\left(\varepsilon_{i}\right)$ and $u_{i, n}=F\left(e_{i, n}\right)=F\left\{Y_{i}-\phi\left(\mathbf{Z}_{i-1}, \boldsymbol{\theta}_{n}\right)\right\}$. With these new definitions, we have $\mathbb{E}_{n}=n^{1 / 2}\left(E_{n}-E\right)$, where $E_{n}(\mathbf{u})=$ $\sum_{i=1}^{n} \mathbf{1}\left(u_{i, n} \leq u_{1}, \ldots, u_{i+m-1, n} \leq u_{m}\right) / n$ and $E(\mathbf{u})=u_{1} \cdots u_{m}$ for all $\mathbf{u}=\left(u_{1}, \ldots, u_{m}\right) \in[0,1]^{m}$. Furthermore, $\tilde{\alpha}_{n}(\mathbf{u})=n^{1 / 2} \times$ $\left\{\sum_{i=1}^{n} \prod_{j=1}^{m} \mathbf{1}\left(U_{i+j-1} \leq u_{j}\right) / n-\prod_{j=1}^{m} u_{j}\right\}$ and

$$
\begin{aligned}
\mathbb{E}(\mathbf{u})=\tilde{\alpha}(\mathbf{u})+\sum_{j=1}^{d} F^{\prime} \circ F^{-1}\left(u_{j}\right)\left(\prod_{\ell>j} u_{\ell}\right) \\
\quad \times E\left\{\dot{\boldsymbol{\phi}}\left(\mathbf{Z}_{j-1}, \boldsymbol{\theta}\right) \prod_{\ell<j} \mathbf{1}\left(U_{\ell} \leq u_{\ell}\right)\right\} \boldsymbol{\Theta},
\end{aligned}
$$

where $\tilde{\alpha}=\alpha\left(F^{-1}, \ldots, F^{-1}\right)$ and $\dot{\boldsymbol{\phi}}(\mathbf{z}, \boldsymbol{\theta})=\nabla_{\boldsymbol{\theta}} \phi(\mathbf{z}, \boldsymbol{\theta})$ is a row vector.

The proof uses asymptotic results from Ghoudi and Rémillard (2004); in particular, the convergence of $\mathbb{E}_{n}$ follows from their theorem 2.4. To check that the assumptions hold, cast the problem in their notation. Set $\mathbf{X}_{i}=\left(Y_{i}, \mathbf{Z}_{i-1}, Y_{i+1}, \mathbf{Z}_{i}, \ldots, Y_{i+m-1}, \mathbf{Z}_{i+m-2}\right) \in \mathfrak{X}=$ $\left([-\infty, \infty]^{1+p}\right)^{\otimes m}$ and $\boldsymbol{\epsilon}_{i}=\left(U_{i}, \ldots, U_{i+m-1}\right)$ for all $i \in \mathbb{N}$. Further, set $\mathbf{X}=\mathbf{X}_{1}$ and $\boldsymbol{\epsilon}=\boldsymbol{\epsilon}_{1}$. Next, for all $\mathbf{x}=\left(y_{1}, \mathbf{z}_{0}, \ldots, y_{m}, \mathbf{z}_{m-1}\right) \in \mathfrak{X}$, define $\mathbb{H}_{n}(\mathbf{x})=\left(\mathbb{H}_{n}^{(1)}(\mathbf{x}), \ldots, \mathbb{H}_{n}^{(m)}(\mathbf{x})\right)$, where for any $j \in\{1, \ldots, m\}$, $\mathbb{H}_{n}^{(j)}(\mathbf{x})=n^{1 / 2}\left\{H_{n}^{(j)}(\mathbf{x})-H^{(j)}(\mathbf{x})\right\}$ with $H^{(j)}(\mathbf{x})=F\left\{y_{j}-\phi\left(\mathbf{z}_{j-1}\right.\right.$, $\boldsymbol{\theta})\}$ and $H_{n}^{(j)}(\mathbf{x})=F\left\{y_{j}-\phi\left(\mathbf{z}_{j-1}, \boldsymbol{\theta}_{n}\right)\right\}$.

Now set $\mathbf{r}=\left(r^{(1)}, \ldots, r^{(m)}\right)$, where for $j \in\{1, \ldots, m\}$ and any $\mathbf{x} \in \mathfrak{X}, r^{(j)}(\mathbf{x})=1+\left\|\dot{\boldsymbol{\phi}}\left(\mathbf{z}_{j-1}, \boldsymbol{\theta}\right)\right\|+\varphi\left(\mathbf{z}_{j-1}, \delta_{0}\right)$, where $\delta_{0}$ is such that $\left\{\boldsymbol{\theta}^{\prime}:\left\|\boldsymbol{\theta}^{\prime}-\boldsymbol{\theta}\right\|<\delta_{0}\right\} \subset \mathcal{O}$, and $\varphi(\mathbf{x}, \delta)=\sup _{\left|\boldsymbol{\theta}^{\prime}-\boldsymbol{\theta}\right|<\delta} \| \dot{\boldsymbol{\phi}}\left(\mathbf{Z}_{0}, \boldsymbol{\theta}^{\prime}\right)-$ $\dot{\boldsymbol{\phi}}\left(\mathbf{Z}_{0}, \boldsymbol{\theta}\right) \|,(\mathbf{x}, \delta) \in \mathfrak{X} \times\left[0, \delta_{0}\right]$.

Finally, let $\mathcal{C}_{r}$ be the set of all $\mathbb{R}^{m}$-valued functions on $\mathfrak{X} \times$ $\mathbb{R}^{d}$ such that $f^{(j)}(\mathbf{x}, \mathbf{a})=-F^{\prime}\left\{y_{j}-\phi\left(\mathbf{z}_{j-1}, \boldsymbol{\theta}\right)\right\} \dot{\boldsymbol{\phi}}\left(\mathbf{z}_{j-1}, \boldsymbol{\theta}\right) \mathbf{a}$ for all $j \in\{1, \ldots, m\}$. Observe that because $F^{\prime}$ is uniformly continuous, there exists a nondecreasing bounded function $c$ on $[0, \infty)$ such that $c(0)=0$ and such that $\left|F(x)-F(y)-(x-y) F^{\prime}(y)\right| \leq$ $|x-y| c(|x-y|)$. Next, $n^{1 / 2}\left|\phi\left(\mathbf{z}, \boldsymbol{\theta}_{n}\right)-\phi(\mathbf{z}, \boldsymbol{\theta})-\nabla_{\boldsymbol{\theta}}(\mathbf{z}, \boldsymbol{\theta}) \boldsymbol{\Theta}_{n}\right| \leq$ $\left\|\boldsymbol{\Theta}_{n}\right\| \varphi\left(\mathbf{z},\left\|\boldsymbol{\theta}_{n}-\boldsymbol{\theta}\right\|\right)$. Setting $d_{n}^{(j)}(\mathbf{x})=\phi\left(\mathbf{z}_{j-1}, \boldsymbol{\theta}_{n}\right)-\phi\left(\mathbf{z}_{j-1}, \boldsymbol{\theta}\right)$, for any $j \in\{1, \ldots, m\}$, it follows that $\left|\mathbb{H}_{n}^{(j)}(\mathbf{x})-f^{(j)}\left(\mathbf{x}, \boldsymbol{\Theta}_{n}\right)\right| \leq$ $n^{1 / 2}\left|d_{n}^{(j)}(\mathbf{x})\right| c\left\{\left|d_{n}^{(j)}(\mathbf{x})\right|\right\}+\left\|\boldsymbol{\Theta}_{n}\right\| \varphi\left(\mathbf{z}_{j-1},\left\|\boldsymbol{\theta}_{n}-\boldsymbol{\theta}\right\|\right)$. So as $n \rightarrow$ $\infty, \sup _{\mathbf{x}}\left|\mathbb{H}_{n}^{(j)}(\mathbf{x})-f^{(j)}\left(\mathbf{x}, \boldsymbol{\Theta}_{n}\right)\right| / r^{(j)}(\mathbf{x}) \stackrel{P}{\rightarrow} 0$ by (11) and the weak convergence of $\boldsymbol{\Theta}_{n}$.

Now if $\left(\alpha_{n}, \boldsymbol{\Theta}_{n}\right) \rightsquigarrow(\alpha, \boldsymbol{\Theta})$ in $\mathcal{D}\left([-\infty, \infty]^{m}\right) \times \mathbb{R}^{d}$ as $n \rightarrow \infty$, and given that condition (11) is satisfied, then $\left(\tilde{\alpha}_{n}, \mathbb{H}_{n}\right)$ converges in $\mathcal{D}\left([0,1]^{m}\right) \times \mathcal{D}\left([-\infty, \infty]^{p}\right)$ to $(\tilde{\alpha}, \mathbb{H})$ as $n \rightarrow \infty$, where $\mathbb{H}^{(j)}(\mathbf{x})=$ $-f^{(j)}(\mathbf{x}, \boldsymbol{\Theta})$ for all $j \in\{1, \ldots, m\}$. Given that $E\left\{\|\mathbf{r}(\mathbf{X})\|^{2}\right\}<\infty$ by hypothesis, lemma 7.2 of Ghoudi and Rémillard (2004) implies that their hypothesis II is verified.

Next, for $f \in \mathcal{C}_{r}$ and $j \in\{1, \ldots, m\}, \mu_{j}\left\{\mathbf{u}, f^{(j)}(\cdot, \mathbf{a})\right\}$ is given by $\mu_{j}\left\{\mathbf{u}, f^{(j)}(\cdot, \mathbf{a})\right\}=F^{\prime} \circ F^{-1}\left(u_{j}\right)\left(\prod_{\ell>j} u_{\ell}\right) E\left\{\dot{\boldsymbol{\phi}}\left(\mathbf{Z}_{j-1}, \boldsymbol{\theta}\right) \times\right.$ $\left.\prod_{\ell<j} \mathbf{1}\left(U_{\ell} \leq u_{\ell}\right)\right\} \mathbf{a}$. Therefore their hypothesis I also is verified, and because $U_{1}, \ldots, U_{m}$ are $\operatorname{Unif}(0,1)$, their hypothesis III is superfluous. Thus we can conclude that $\mathbb{E}_{n} \rightsquigarrow \tilde{\mathbb{E}}$ in $\mathcal{D}\left([0,1]^{m}\right)$ as $n \rightarrow \infty$, where $\mathbb{E}$ has representation (B.1).

\section{[Received April 2006. Revised July 2007.]}

\section{REFERENCES}

Berkes, I., and Horváth, L. (2003), "Limit Results for the Empirical Process of Squared Residuals in GARCH Models," Stochastic Processes and Their Applications, 105, 271-298.

Bilodeau, M., and Lafaye de Micheaux, P. (2005), "A Multivariate Empirical Characteristic Function Test of Independence With Normal Marginals," Journal of Multivariate Analysis, 95, 345-369.

Box, G. E. P., Jenkins, G. M., and Reinsel, G. C. (1994), Time Series Analysis (3rd ed.), Englewood Cliffs, NJ: Prentice-Hall.

Brock, W. A., Dechert, W. D., LeBaron, B., and Scheinkman, J. A. (1996), "A Test for Independence Based on the Correlation Dimension," Economic Review, 15, 197-235.

Brock, W. A., Hsieh, D. A., and LeBaron, B. (1991), Nonlinear Dynamics, Chaos, and Instability: Statistical Theory and Economic Evidence, Cambridge, MA: MIT Press.

Brockwell, P. J., and Davis, R. A. (1991), Time Series: Theory and Methods (2nd ed.), New York: Springer.

Chatterjee, S., and Yilmaz, M. R. (1992), "Chaos, Fractals and Statistics," Statistical Science, 7, 49-68.

Delgado, M. A. (1996), "Testing Serial Independence Using the Sample Distribution Function," Journal of Time Series Analysis, 17, 271-285.

Dufour, J.-M., and Roy, R. (1985), "Some Robust Exact Results on Sample Autocorrelations and Tests of Randomness," Journal of Economics, 29, 257-273.

Ferguson, T. S., Genest, C., and Hallin, M. (2000), "Kendall's Tau for Serial Dependence," The Canadian Journal of Statistics, 28, 587-604.

Genest, C., and Rémillard, B. (2004), "Tests of Independence or Randomness Based on the Empirical Copula Process," Test, 13, 335-369.

Genest, C., Quessy, J.-F., and Rémillard, B. (2002), "Tests of Serial Independence Based on Kendall's Process," The Canadian Journal of Statistics, 30, $441-461$.

Ghoudi, K., Kulperger, R. J., and Rémillard, B. (2001), “A Nonparametric Test of Serial Independence for Time Series and Residuals," Journal of Multivariate Analysis, 79, 191-218.

Ghoudi, K., and Rémillard, B. (2004), "Empirical Processes Based on PseudoObservations. II. The Multivariate Case," in Asymptotic Methods in Stochastics, Providence, RI: American Mathematical Society, pp. 381-406.

Hallin, M., and Jurečková, J. (1999), "Optimal Tests for Autoregressive Models Based on Autoregression Rank Scores," The Annals of Statistics, 27, 1385-1414.

Hallin, M., and Puri, M. L. (1992), "Rank Tests for Time Series Analysis: A Survey," in New Directions in Time Series Analysis, Part I, eds. D. R. Brillinger, E. Parzen, and M. Rosenblatt, New York: Springer, pp. 111-153. (1994), "Aligned Rank Tests for Linear Models With Autocorrelated Error Terms," Journal of Multivariate Analysis, 50, 175-237.

Hallin, M., Ingenbleek, J.-F., and Puri, M. L. (1985), "Linear Serial Rank Tests for Randomness Against ARMA Alternatives," The Annals of Statistics, 13, $1156-1181$.

(1987), "Linear and Quadratic Serial Rank Tests for Randomness Against Serial Dependence," Journal of Time Series Analysis, 8, 409-424.

Hong, Y. (1999), "Hypothesis Testing in Time Series via the Empirical Characteristic Function: A Generalized Spectral Density Approach," Journal of the American Statistical Association, 94, 1201-1220.

(2000), "Generalized Spectral Tests for Serial Dependence," Journal of the Royal Statistical Society, Ser. B, 62, 557-574.

Hong, Y., and White, H. (2005), "Asymptotic Distribution Theory for Nonparametric Entropy Measures of Serial Dependence," Econometrica, 73, 837-901.

Kočenda, E. (2001), "An Alternative to the BDS Test: Integration Across the Correlation Integral," Econometric Review, 20, 337-351.

Kočenda, E., and Briatka, Ľ. (2005), "Optimal Range for the iid Test Based on Integration Across the Correlation Integral," Econometric Review, 24, 265-296.

Ljung, G. M., and Box, G. E. P. (1978), "On a Measure of Lack of Fit in Time Series Models," Biometrika, 65, 297-303.

Moran, P. A. P. (1948), "Some Theorems on Time Series, II: The Significance of the Serial Correlation Coefficient," Biometrika, 35, 255-260.

Robinson, P. M. (1991), "Consistent Nonparametric Entropy-Based Testing," Review of Economic Studies, 58, 437-453.

Skaug, H. J., and Tjøstheim, D. (1993), "A Nonparametric Test of Serial Independence Based on the Empirical Distribution Function," Biometrika, 80, $591-602$.

Tong, H., and Lim, K. S. (1980), "Threshold Autoregression, Limit Cycles and Cyclical Data," Journal of the Royal Statistical Society, Ser. B, 42, 245-292. 